\title{
Infrared radiative transfer in atmospheres of Earth-like planets around F, G, K, and M stars
}

\section{Thermal emission spectra influenced by clouds}

\author{
M. Vasquez ${ }^{1}$, F. Schreier ${ }^{1}$, S. Gimeno García ${ }^{1,2}$, D. Kitzmann ${ }^{3}$, B. Patzer ${ }^{3}$, H. Rauer ${ }^{3,4}$, and T. Trautmann ${ }^{1}$ \\ ${ }^{1}$ DLR - Deutsches Zentrum für Luft- und Raumfahrt, Institut für Methodik der Fernerkundung, Oberpfaffenhofen, 82234 Weßling, \\ Germany \\ e-mail: mayte.vasquez@dlr.de \\ 2 TUM - Technische Universität München, Lehrstuhl für Methodik der Fernerkundung, Arcisstr. 21, 80333 München, Germany \\ 3 TUB - Technische Universität Berlin, Zentrum für Astronomie und Astrophysik, Hardenbergstr. 36, 10623 Berlin, Germany \\ ${ }^{4}$ DLR - Deutsches Zentrum für Luft- und Raumfahrt, Institut für Planetenforschung, Rutherfordstr. 2, 12489 Berlin, Germany
}

Received 15 October 2012 / Accepted 24 May 2013

\begin{abstract}
Context. Clouds play an important role in the radiative transfer of planetary atmospheres because of the influence they have on the different molecular signatures through scattering and absorption processes. Furthermore, they are important modulators of the radiative energy budget affecting surface and atmospheric temperatures.

Aims. We present a detailed study of the thermal emission of cloud-covered planets orbiting F-, G-, K-, and M-type stars. These Earth-like planets include planets with the same gravity and total irradiation as Earth, but can differ significantly in the upper atmosphere. The impact of single-layered clouds is analyzed to determine what information on the atmosphere may be lost or gained. The planetary spectra are studied at different instrument resolutions and compared to previously calculated low-resolution spectra.

Methods. A line-by-line molecular absorption model coupled with a multiple scattering radiative transfer solver was used to calculate the spectra of cloud-covered planets. The atmospheric profiles used in the radiation calculations were obtained with a radiativeconvective climate model combined with a parametric cloud description.

Results. In the high-resolution flux spectra, clouds changed the intensities and shapes of the bands of $\mathrm{CO}_{2}, \mathrm{~N}_{2} \mathrm{O}, \mathrm{H}_{2} \mathrm{O}, \mathrm{CH}_{4}$, and $\mathrm{O}_{3}$. Some of these bands turned out to be highly reduced by the presence of clouds, which causes difficulties for their detection. The most affected spectral bands resulted for the planet orbiting the F-type star. Clouds could lead to false negative interpretations for the different molecular species investigated. However, at low resolution, clouds were found to be crucial for detecting some of the molecular bands that could not be distinguished in the cloud-free atmospheres. The $\mathrm{CO}_{2}$ bands were found to be less affected by clouds. Radiation sources were visualized with weighting functions at high resolution.

Conclusions. Knowledge of the atmospheric temperature profile is essential for estimating the composition and important for avoiding false negative detection of biomarkers, in both cloudy and clear-sky conditions. In particular, a pronounced temperature contrast between the ozone layer and surface or cloud is needed to detect the molecule. Fortunately, the $\mathrm{CO}_{2}$ bands allow temperature estimation from the upper stratosphere down to the troposphere even in the presence of clouds.
\end{abstract}

Key words. astrobiology - radiative transfer - techniques: spectroscopic - planets and satellites: atmospheres infrared: planetary systems

\section{Introduction}

The search for life on extrasolar planets is of main interest in exoplanetary sciences. More than 880 exoplanets have been detected so far with a few thousand candidates that have been gathered by NASA's Kepler mission and are waiting for confirmation (Batalha et al. 2013). Some of these planets have been estimated as similar in size to the terrestrial planets (e.g., Fressin et al. 2011; Gilliland et al. 2013; Barclay et al. 2013), and several studies have identified exoplanets within the habitable zone (Lovis et al. 2006; Udry et al. 2007; Borucki et al. 2011; Koch et al. 2012). In terms of mass and radius, about ten planets with $0.5<M / M_{\oplus}<2.0$ and roughly 15 with $0.8<R / R_{\oplus}<1.3$ have been found. Future missions (e.g., ESA's PLATO and EChO) dedicated to the search and characterization of exoplanets will further raise the probability of finding planets that are similar to Earth.
The study of potential habitability concentrates on Earth-like planets because Earth is the only known example of a planet sustaining life. Here, "Earth-like" is used to characterize planets with the same mass, radius (hence gravity), and total irradiation level. Moreover, nitrogen and oxygen abundances are identical to Earth today. However, due to the different spectral distribution of the stellar radiation, temperature and water can be significantly different in the upper atmosphere.

Remote sensing offers the only way to determine the planet's atmospheric state and composition. Therefore, it is essential to study signatures of its constituents under varying conditions in order to understand possible changes in the spectrum. Along with molecular constituents, planetary atmospheres may have particle condensates forming clouds that influence chemistry, dynamics, and radiation. Therefore, for remote sensing of planetary atmospheres, it is important to analyze the impact of clouds on the spectra. 
Clouds can inhibit the release of energy (warming greenhouse effect), and they can reflect the incoming stellar light back to space (cooling albedo effect). Since clouds affect atmospheres, they can also generate false interpretations of spectral signatures by masking the radiation arising from layers below the cloud deck.

Observations of Earth and Venus have shown that clouds influence the radiation. Hearty et al. (2009) analyzed infrared (IR) satellite spectra of Earth under clear and cloudy conditions and showed that clouds led to a reduction of the intensity and signatures, raising the difficulty of detecting some of the molecules, especially at low spectral resolutions. Venus is probably the most drastic example for demonstrating how a dense cloud deck can significantly affect the spectrum (e.g. Haus \& Arnold 2010).

For stellar objects outside our solar system, Ackerman \& Marley (2001), De Kok et al. (2011) and Burrows et al. (2011) studied the spectra of brown dwarfs, including clouds in the radiative transfer modeling. Cushing et al. (2008) and Witte et al. (2011) showed that clouded atmospheres lead to better agreement with the observations of brown dwarfs. Also, Helling et al. (2008) analyzed the cloud particles on these bodies in detail.

Clouds are also studied for interpreting hot-Jupiter exoplanet observations. For HD 189733, Pont et al. (2008) and Sing et al. (2011) attributed missing molecular absorption lines to haze composed of sub-micron particles, and Huitson et al. (2012) explained the featureless spectrum by scattering particles and estimated their composition. Charbonneau et al. (2002) analyzed the exoplanet HD 209458 and proposed a high cloud in its atmosphere.

Ground-based observations of the super-Earth GJ1214b (Bean et al. 2010) have also shown a nearly flat transmission spectrum in the visible. Radiative transfer modeling under different atmospheric scenarios indicated that the lack of spectral features could be attributed to high clouds. Berta et al. (2012) varied the cloud optical thickness to fit the GJ1214b observations.

Biosignatures (i.e., the spectral footprints by atmospheric molecules that could indicate the presence of life) of clear-sky planets have been explored by several groups for different spectral regions by radiative transfer modeling. Des Marais et al. (2002) modeled the spectra of a terrestrial planet in order to investigate the different molecular signatures in the visible to IR regions that could be useful when charaterizing atmospheres in the search for habitable planets. Schindler \& Kasting (2000) and Selsis et al. (2002) have addressed the false-positive detection of biosignatures using NASA's Terrestrial Planet Finder and ESA's Darwin, respectively. Segura et al. (2003, 2005) modeled Earth-like planets around different type of stars (i.e. F, G, $\mathrm{K}$, and $\mathrm{M}$ ) in order to determine observable spectral signatures. Grenfell et al. (2011) compared the different signatures from a modern and proterozoic epoch Earth. Rauer et al. (2011) studied the transmission and emission spectra of super-Earth atmospheres around M-type stars with emphasis on the atmospheric chemistry. These studies survey the different biosignatures and determined species observable in cloud-free spectra.

Several studies have also addressed the detection of biosignatures in the presence of clouds. Des Marais et al. (2002) discussed the Earth spectra by introducing absorbing and emitting cloud layers at different atmospheric altitudes. Kaltenegger et al. (2007) included clouds on Earth for different geological epochs assuming weighted averages for low-, medium-, and high-level clouds. In contrast to these studies, Tinetti et al. (2006a,b) used a more sophisticated radiative transfer including multiple scattering to study the disk-averaged spectra of Earth for low-, medium-, and high-level clouds. In none of these studies a thermodynamically consistent atmosphere computed with a proper treatment of cloud radiative forcing was considered.

In contrast, our work is founded on thermodynamically consistent surface and atmospheric temperatures and water vapor concentrations in cloudy atmospheres of Earth-like planets around main sequence stars (see Kitzmann et al. 2010). The atmospheric model sustains a more accurate treatment of the clouds radiative forcing that affects the radiation budget of a planet by including water and ice particles scattering and absorption.

Based on these modeling studies, Kitzmann et al. (2011) presented the first analysis of IR spectra (albeit at low resolution due to the correlated-k approach) in thermodynamically consistent cloudy Earth-like exoplanetary atmospheres. They found that clouds reduced the strength of the molecular bands, especially in the planet orbiting the F star. In addition, they concluded that no absorption features could be distinguished for high cloud coverages in the modeled spectra of the F-star planet. However, an identification and a detailed analysis of the main molecular bands found could not be performed owing to model flexibility and spectral-resolution limitations.

We overcome these limitations by using a flexible line-byline multiple-scattering radiative transfer model that allows us to make the first rigorous study of the important molecular bands in the thermal IR with different cloudy conditions. The thermal emission spectra of Earth-like planets are calculated by taking the effects of clouds on planets orbiting different types of main sequence host stars into account: F2V, G2V, K2V, and M3.5V. The atmospheres for these planets are built upon the radiativeconvective atmospheric model of Kitzmann et al. (2010). The planetary spectra for cloud-free atmospheres have been analyzed in detail in Vasquez et al. (2013b, henceforth called Paper I), which contains a description of the main biomarkers found in the thermal region that are investigated for habitability. These signatures are also investigated in detail in this work but now including clouds. Accordingly, this study also extends the investigations presented by Kitzmann et al. (2011).

Our objective is to provide a comprehensive analysis of IR spectra at high resolution and to explore the molecular bands of cloud covered Earth-like planets. This study, along with Paper I, may serve as a guide in the future phase of terrestrial exoplanet observations in which it might be possible to directly analyze of observed planetary spectra.

A description of the radiative transfer model is found in Sect. 2. Details of the atmospheric scenarios and cloud optical properties used as input are also given here. An analysis of the modeled spectra at different resolutions is given in Sect. 3 along with comparisons of the low-resolution spectra to the previous study of Kitzmann et al. (2011). Assumptions, limitations, and possible extensions of our study are discussed in Sect. 4, and a summary is given in Sect. 5.

\section{Models, data, and scenarios}

\subsection{Radiative transfer model}

The radiative transfer model GARLIC (Generic Atmospheric Radiation Line-by-line Infrared Code, the Fortran 90 version of the MIRART/SQuIRRL model, Schreier \& Schimpf 2001) is used to calculate line-by-line IR spectra of Earth-like planets (Paper I). To take multiple scattering events into account, GARLIC has been coupled with the radiative transfer solver DISORT (Discrete Ordinates Radiative Transfer), which solves 
the radiative transfer for a one-dimensional atmosphere in a multi-layered plane parallel medium (Stamnes et al. 1988).

GARLIC takes the pressure and temperature profiles of the planet as input, together with the atmospheric composition and the cloud optical properties (optical depth, single scattering albedo, and asymmetry parameter). These input data are taken from Kitzmann et al. (2010) and discussed in the following sections. Molecular optical properties are calculated in GARLIC using HITRAN 2008 spectroscopy data (Rothman et al. 2009) and the CKD water continuum (Clough et al. 1989), which then passes the total atmospheric optical depths with particle and molecular contributions to DISORT. GARLIC was successfully used with a single scattering approximation to model the Venus spectra measured by SCIAMACHY considering a haze region above the planet main cloud deck (Vasquez et al. 2013a).

Radiative transfer in the atmosphere is determined by absorption, emission, and scattering. Taking these processes into account, the formal solution to the radiative transfer equation used in GARLIC, is given by (e.g., Zdunkowski et al. 2007)

$\mathcal{I}(v)=I_{\mathrm{o}}(v) \mathrm{e}^{-\tau(v)}+\int_{0}^{\tau} J\left(\tau^{\prime}\right) \mathrm{e}^{-\tau^{\prime}} \mathrm{d} \tau^{\prime}$,

where $\mathcal{I}_{o}$ is the incident intensity at the boundary, originating at the planet's surface, and $\mathcal{T}=\mathrm{e}^{-\tau(v)}$ represents the transmission. The optical depth, $\tau$, is the integral along the photon path of the volume extinction coefficient,

$\tau=\int \kappa \mathrm{d} s=\tau^{\text {part }}+\tau^{\mathrm{mol}}$

where the optical depth is comprised of the optical depths of the cloud particles and molecules.

Since contributions from molecular scattering (Rayleigh) are neglected in the thermal region, the source term in Eq. (1) includes only thermal emission and scattering by particles,

$J=\left(1-\omega_{o}\right) B(v, T)+\omega_{o} \int_{0}^{4 \pi} P(\cos \Theta) \mathcal{I}\left(\Omega^{\prime}\right) \mathrm{d} \Omega^{\prime}$,

where the thermal emission source is determined by the Planck function, $B$ at temperature $T$, and single scattering albedo, $\omega_{o}$, which represents the fraction of light that undergoes a scattering event. Accordingly, $\left(1-\omega_{o}\right)$ represents the fraction of absorption. The scattering source term depends on the (normalized) scattering phase function $P(\cos \Theta)$ (where $\Theta=\Omega \cdot \Omega^{\prime}$ is the scattering angle), which represents the probability of light being scattered by an angle between $\Theta$ and $\Theta+\mathrm{d} \Theta$.

The total single scattering albedo is given by $\omega_{0}=$ $\omega_{o}^{\text {part }} \tau^{\text {part }} / \tau$, and the Henyey-Greenstein phase function at the cloud layer required to solve (3) is

$P_{\mathrm{HG}}(\cos \Theta)=\frac{1}{4 \pi} \frac{1-g^{2}}{\left(1+g^{2}-2 g \cos \Theta\right)^{3 / 2}}$.

The phase function depends on the asymmetry parameter, $g$, which describes the amount of backward and forward scattering, ranging from -1 (totally backward) to 1 (totally forward).

The planetary spectra of a distant object, such as exoplanets, is customarily expressed in flux rather than in intensity, which is obtained through the hemispherical integration of the outgoing intensities. The spectra were modeled using an ideal instrument at different resolutions (low, medium, and high) represented by respective convolutions with Gaussian response functions. Here, we use the word "ideal" to denote an instrument without any noise contamination (for a detailed discussion see Rauer et al. 2011 or von Paris et al. 2011), so the only instrumental effect is due to finite spectral resolution.
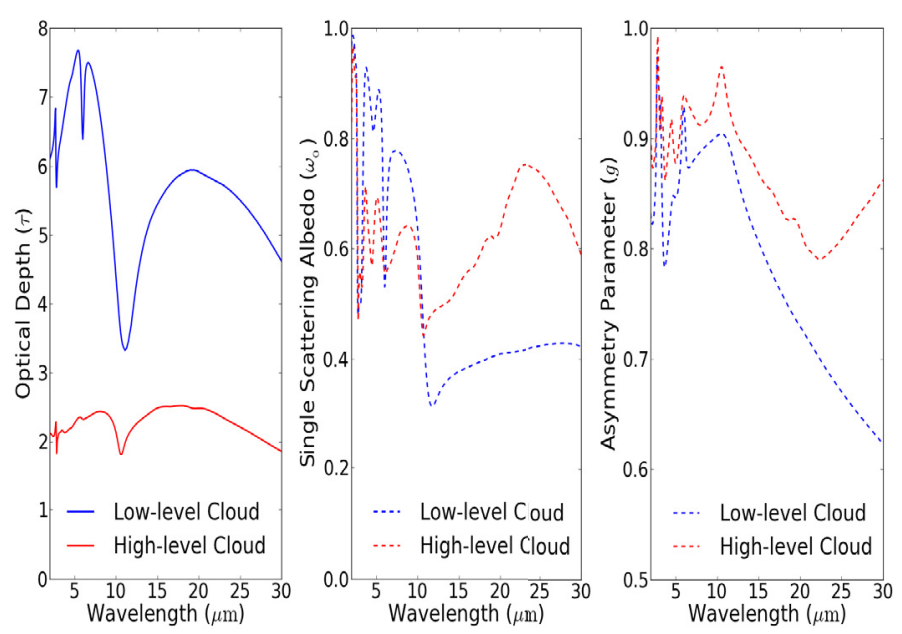

Fig. 1. Cloud particles optical depth, single scattering albedo and asymmetry parameter for low- and high-level clouds. For more details see Kitzmann et al. (2010).

\subsection{Cloud optical properties}

The parametric cloud description used in the atmospheric model is based on observations of clouds in the Earth's atmosphere. In particular, two cloud layers are considered: a low-level liquid water and a high-level water ice cloud. The optical properties of the low- and high-level clouds provided by Kitzmann et al. (2010) were used in the multiple scattering calculations and are shown in Fig. 1. The particle size distribution of the low-level cloud is assumed to be log-normal with measured parameters (e.g., mean particle radius and standard deviation) taken from Kokhanovsky (2006). For the high-level ice cloud, Kitzmann et al. (2010) used the power law size distribution published by Heymsfield \& Platt (1984) which were based on in-situ measurements. Other average cloud properties, such as the cloud top temperature and pressure, as well as their optical depth, have been taken from the long-term satellite-based measurements within the International Satellite Cloud Climatology Project (ISCCP; Rossow \& Schiffer 1999). Kitzmann et al. (2010) iteratively adjusted the altitude of each cloud layer to match the corresponding observed pressures (i.e., cloud heights were not fixed) and used Mie theory to obtain the size-integrated scattering and absorption cross-sections and asymmetry parameters (Bohren \& Huffman 1983). The ice crystals were assumed to be composed of hexagonal columns, and an equivalent sphere approach was used.

The low-level cloud presents a larger optical depth than the high-level cloud due to the larger particle number density. The spectral dependency of the optical depth is different for the water and ice clouds.

The single scattering albedo controls the relative importance of scattering against absorption contributions to the optical depth. Values close to unity mean that the cloud-light interaction is mostly scattering, whereas values close to zero indicate that the interaction is mostly absorption. For the low-level cloud, most of the light at short wavelengths is scattered predominantly in the forward direction (Fig. 1), while at longer wavelengths (above $11 \mu \mathrm{m}$ ) most of the light is absorbed. A different situation occurs for the high-level cloud, which scatters a larger portion of the light around $25 \mu \mathrm{m}$, while at shorter wavelengths $(4-10 \mu \mathrm{m})$ absorption and scattering are of comparable magnitudes. 


\subsection{Planetary scenarios}

The equilibrium atmospheric profiles were calculated with a one-dimensional radiative-convective climate model that takes the cloud radiative forcing into account (Kitzmann et al. 2010) and is based on the photochemical model of Kasting et al. (1984); Segura et al. (2003); Grenfell et al. (2007). The distance between the planets and their host stars were scaled in order for them to receive the same amount of stellar energy that Earth receives at the top of its atmosphere, ToA.

The pressure-temperature profiles of atmospheres for different coverages of low- (top) and high-level (bottom) clouds depending on planet and cloud are illustrated in Fig. 2, where the impact produced by these two different cloud compositions and coverages on the atmospheric temperatures can be seen (Kitzmann et al. 2010). Different molecular concentrations profiles exist for different planet types (Grenfell et al. 2007). Nevertheless, to maintain consistency with Paper I and Kitzmann et al. (2011), we used the Kitzmann et al. (2010) atmospheric scenarios; that is, temperature, pressure and $\mathrm{H}_{2} \mathrm{O}$ concentrations depending on planet and cloud.

In the lower atmosphere the abundances of $\mathrm{H}_{2} \mathrm{O}$ in the gas phase are not controlled by chemistry, but rather by convective transport of $\mathrm{H}_{2} \mathrm{O}$ from the surface up to the tropopause. The relative humidity in the troposphere is determined by the empirical distribution of Manabe \& Wetherald (1967), which implicitly assumes that any supersaturated $\mathrm{H}_{2} \mathrm{O}$ is removed from the vapor phase by condensation to form cloud particles.

The atmospheric profiles of the major chemical species were obtained with a detailed photochemical model (Grenfell et al. 2007) representing modern Earth. Isoprofiles are used for the well mixed gases $\mathrm{N}_{2}, \mathrm{O}_{2}$, and $\mathrm{CO}_{2}$. The profiles of $\mathrm{H}_{2} \mathrm{O}$ (in the upper atmosphere), $\mathrm{CH}_{4}, \mathrm{O}_{3}$, and $\mathrm{N}_{2} \mathrm{O}$ were derived for the modern Earth. This atmospheric composition is assumed for all calculations, thereby neglecting any change in the processes influencing the chemical composition of the planetary atmospheres (see Kitzmann et al. 2010, for details).

The profiles of the planets around $\mathrm{K}$ and $\mathrm{M}$ stars indicate that the different low cloud coverages have a greater impact on their stratospheres than in the ones around the $F$ and $G$ stars. In the presence of the high cloud, the stratospheric temperatures in all the planets are not affected much by the different cloud coverages. However, this type of cloud raises the tropospheric temperatures of the planets, while the low-level cloud produces the opposite effect.

The planetary surface temperature is also strongly influenced by clouds. The low-level cloud produces a cooling effect, while the high-level cloud produces a warming effect. In some of the coverage cases of the low-level cloud, the temperatures even drop below the freezing point of water. In the presence of the high-level cloud for all the different cloud coverages, the temperatures remain below the boiling point of water (see Kitzmann et al. 2010, for more details).

\section{Infrared spectra in the presence of clouds}

Clouds can have an impact on the shape and the strength of the spectral absorption bands, which may cause false negative results. This impact can be caused by a temperature difference between the cloud layer (major emitting source in cloudy atmospheres) and the surface of the planet (major emitting source in cloud-free atmospheres, see Paper I). Also, the thickness of the cloud plays a role in the degree of modification of the spectra. Optically thick clouds have a larger impact on the radiation
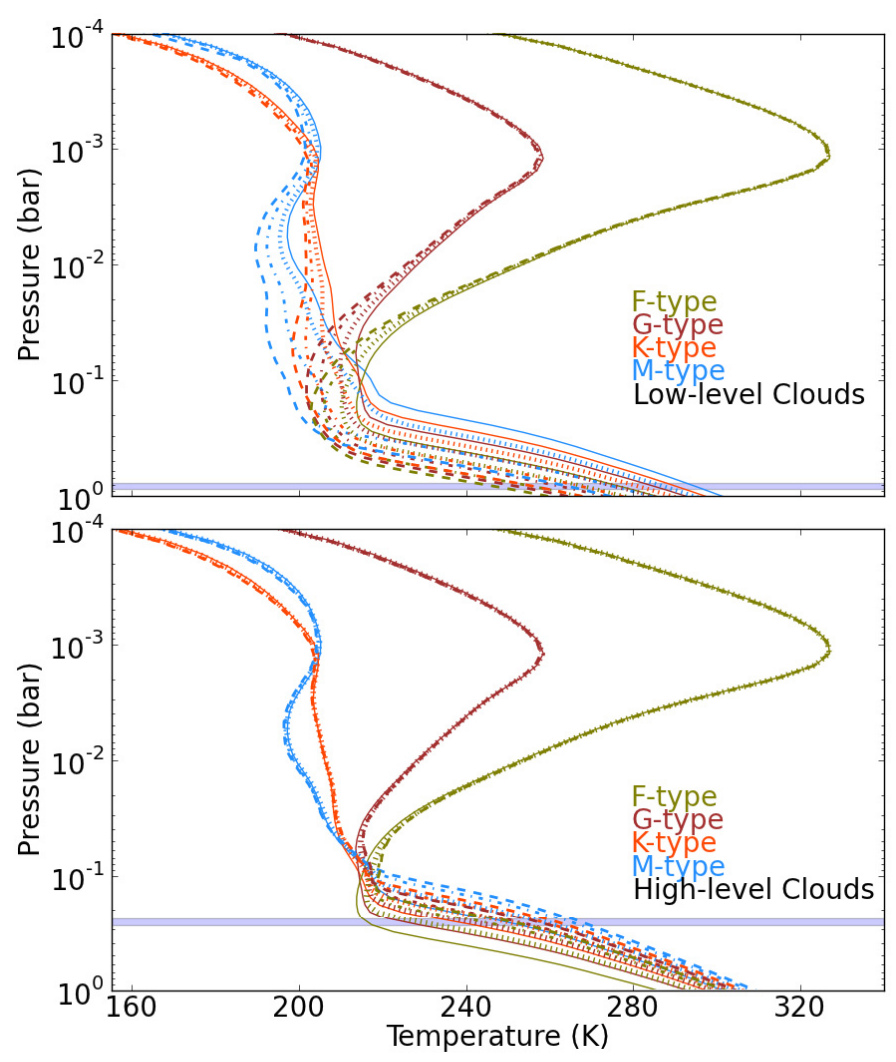

Fig. 2. Pressure-temperature profiles influenced by low and high clouds for four different coverages (Kitzmann et al. 2010): 0\% (solid lines), $30 \%$ (dotted), $70 \%$ (dash-dot), and 100\% (dashed). Planets are modeled around typical F (olive green), G (brown), K (orange), and M (blue) star types. The location of the low- (top) and high-level (bottom) clouds is also illustrated.

arising from layers below the cloud deck than thinner clouds owing to the greater strength of absorption and number of scattering events. Low- and high-level clouds affect the spectra in different ways since their corresponding temperatures (Fig. 2) and optical properties (Fig. 1) differ considerably. In the context of this paper, false negatives denote the failure to identify signatures of molecules present in the planetary atmospheres owing to the impact that clouds have in the radiative transfer. The opposite effect, known as false positive results, could not be found in the planetary spectra.

\subsection{High-resolution spectra}

The spectra of Earth-like planets influenced by four different cloud-cover fractions were analyzed, namely $0 \%, 30 \%, 70 \%$, and $100 \%$, with emphasis on the $\mathrm{CO}_{2}(4.3$ and $15 \mu \mathrm{m}), \mathrm{N}_{2} \mathrm{O}$ $(4.5 \mu \mathrm{m}), \mathrm{H}_{2} \mathrm{O}(6.3 \mu \mathrm{m}), \mathrm{CH}_{4}(7.7 \mu \mathrm{m})$, and $\mathrm{O}_{3}(9.6 \mu \mathrm{m})$ bands. The cloud fraction characterizes the area of the planet covered by clouds. Cloud-free $(0 \%)$ and complete cloud $(100 \%)$ covered planets were chosen as extreme cases and $30 \%$ and $70 \%$ as intermediate cases. For cloud-free atmospheres, a detailed analysis of the physical processes with an explanation of the shape and strength of the spectral appearance is provided in Paper I. In the following, we emphasize cloud related aspects.

Figure 3 illustrates the planetary spectra in flux units at a resolution of $3000(R=\lambda / \Delta \lambda)$. In accordance with Paper I, molecular bands are shown by separate for a smaller range of wavelength in the figures to follow in equivalent brightness 
M. Vasquez et al.: IR radiative transfer in atmospheres of Earth-like planets influenced by clouds
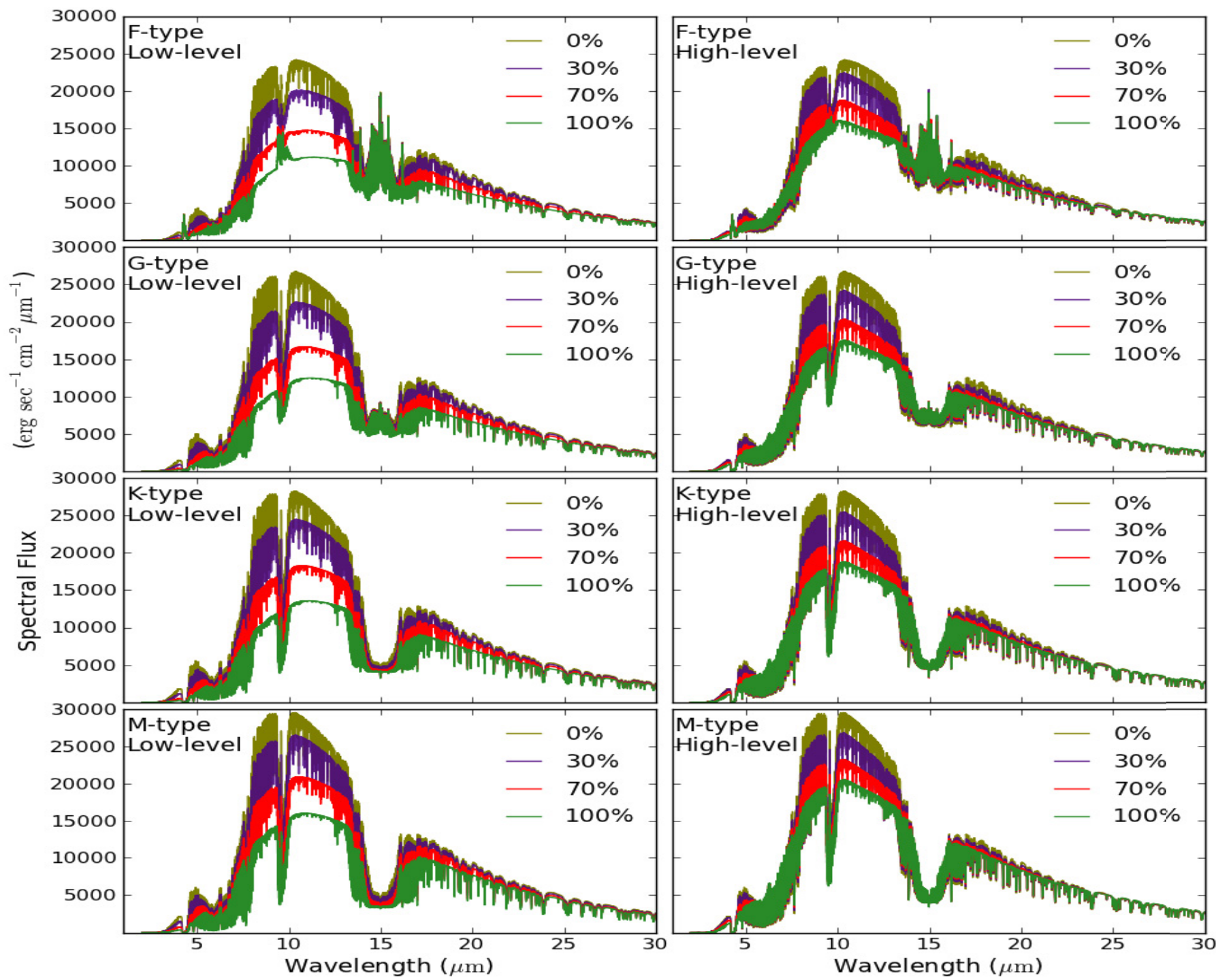

Fig. 3. Thermal emission spectra of Earth-like planets around four different types of stars for two cloud conditions with four different percent coverages.

temperature, essentially the inverse of the Planck function, $T_{\mathrm{B}}=$ $B^{-1}$ (e.g., Hanel et al. 2003). At a given monochromatic wavelength, the brightness temperature is close to the atmospheric temperature in the altitude regime providing the dominant contribution to the upwelling radiation.

This mapping of spectral regions to altitudes is indicated by weighting functions (see Paper I for a more detailed discussion), defined by the partial derivatives $\partial \mathcal{T}(v, z) / \partial z$ of the transmission along the different altitudes (Fig. 4). These reveal the contributions arising from each atmospheric layer to ToA. The weighting functions of the F-star planet are shown for the cases of $100 \%$ low-level liquid water and high-level ice water clouds (see Paper I for the corresponding cloud-free atmosphere). In the presence of the high-level cloud, contributions from below the cloud can be seen at the ToA, while contributions from below are almost completely blocked with the low-level cloud. This is mainly because the optical depth of the liquid water cloud is higher than the one of the ice water cloud - typically for a terrestrial boundary layer compared to cirrus clouds (see Kitzmann et al. 2010 for detailed information about the cloud scenarios).

For most wavelengths, the main contributing layer to the radiation flux is at the atmospheric altitude at or near the cloud deck (see Fig. 4), which is at a lower temperature than the planet's surface. This results in a decrease of the planetary emission flux, since their corresponding blackbody curves are lower than in the cloud-free atmosphere. Accordingly, the planetary emission flux is reduced the most in the $100 \%$ coverage for both cloudy conditions.

In the atmospheric window, between about 800 and $1250 \mathrm{~cm}^{-1}(8-12.5 \mu \mathrm{m})$, for cloud-free conditions, the atmosphere is nearly transparent but not in the presence of clouds. The temperature difference between the low-level cloud and the surface is less than the difference between the surface and the high-level cloud. Figure 5 depicts the emission spectra of the planet F star under different cloudy conditions with the respective blackbody curves corresponding to the surface ( $\left.T_{\text {surf }}\right)$, cloud-level temperature $\left(T_{\text {cld }}\right)$ and curves matching the spectra $\left(T_{\mathrm{mt}}\right)$ best. For low-level clouds with a high percent coverage, the brightness temperature observed at the ToA is related to the cloud-level temperature due to the large optical depth (see Fig. 1). Nevertheless, in the presence of high clouds, this brightness temperature is related to layers found below the cloud, since the optical depth is not so large and only partly blocks these layers. 

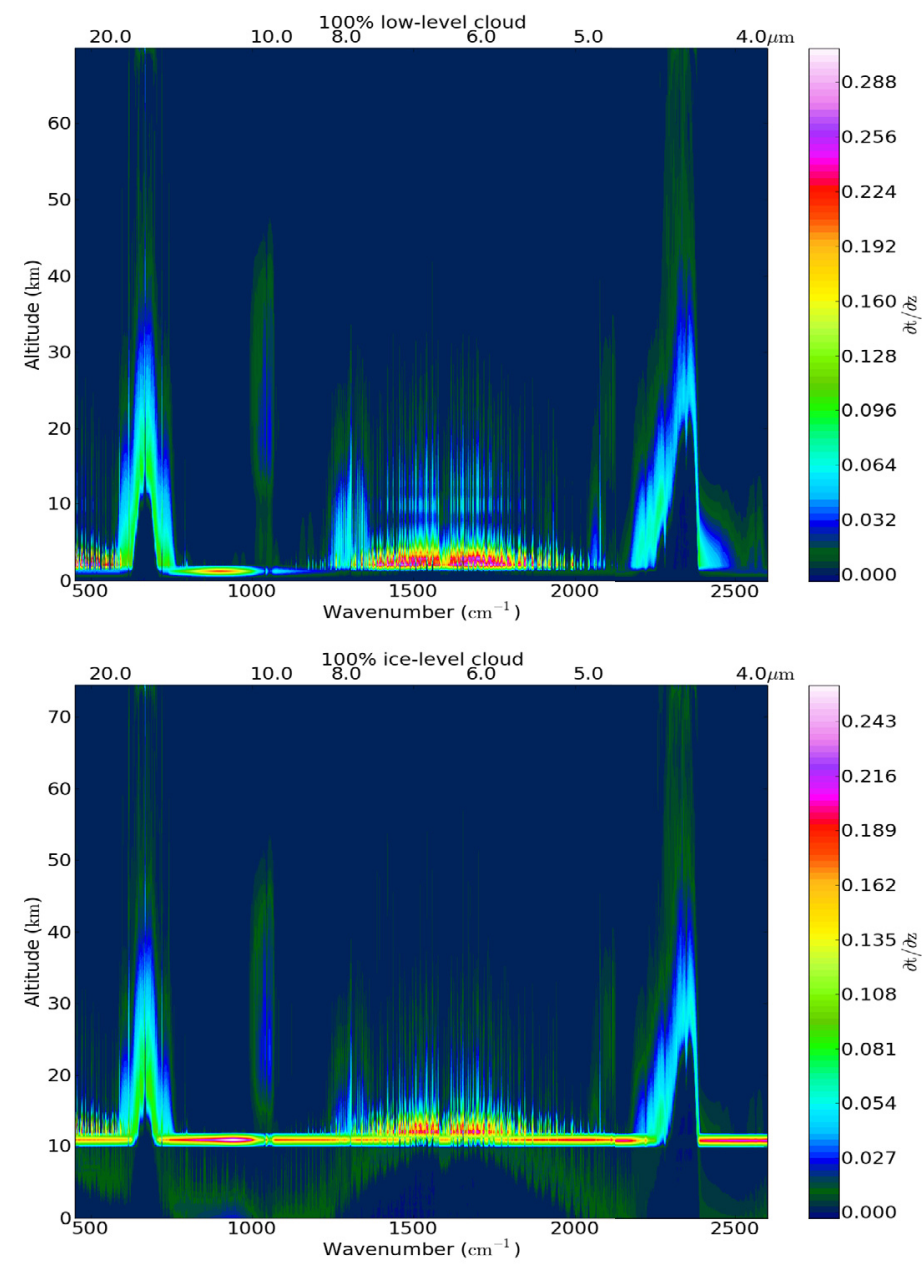

Fig. 4. Weighting functions for the F-star planet influenced by the $100 \%$ low and high-level clouds at a resolution of 3000.

For the atmospheres with high-level clouds, the cloud layer temperature differs considerably from the surface temperature, which prevents the use of the atmospheric window to infer the planet's surface temperature. For the $30 \%$ low-level cloud, one can still obtain a reasonable estimate of the surface temperature.

\subsubsection{Carbon dioxide}

Carbon dioxide is a uniformly mixed gas throughout the atmosphere with dominant features at $4.3 \mu \mathrm{m}$ and $15 \mu \mathrm{m}$ (see Paper I for more details on this and the following molecules). The relative depth of the $4.3 \mu \mathrm{m} \mathrm{CO} 2$ band is reduced by the presence of clouds in all planetary cases. As already mentioned in Paper I for cloud-free atmospheres, the absorption optical depths (Eq. (2)) in the center of the $4.3 \mu \mathrm{m} \mathrm{CO} 2$ band are very large and, consequently, only radiation emitted in the upper stratosphere reaches the ToA. It was also mentioned that in the planet orbiting the F star, the $\mathrm{CO}_{2}$ bands appear as an emission spectrum due to a temperature inversion found in the stratosphere.

Figures 4 and 6 show that in the F-star planet, the different cloud coverages have almost no influence on the central region of the band, between 4.2-4.4 $\mu \mathrm{m}$. The same occurs in the G-star planet. However, in the $\mathrm{K}$ - and $\mathrm{M}$-star planets, the different coverages have a greater impact on the band. Figure 2 can be used to relate the atmospheric temperatures to the brightness temperature spectrum. The low-level cloud leads to a stronger cooling

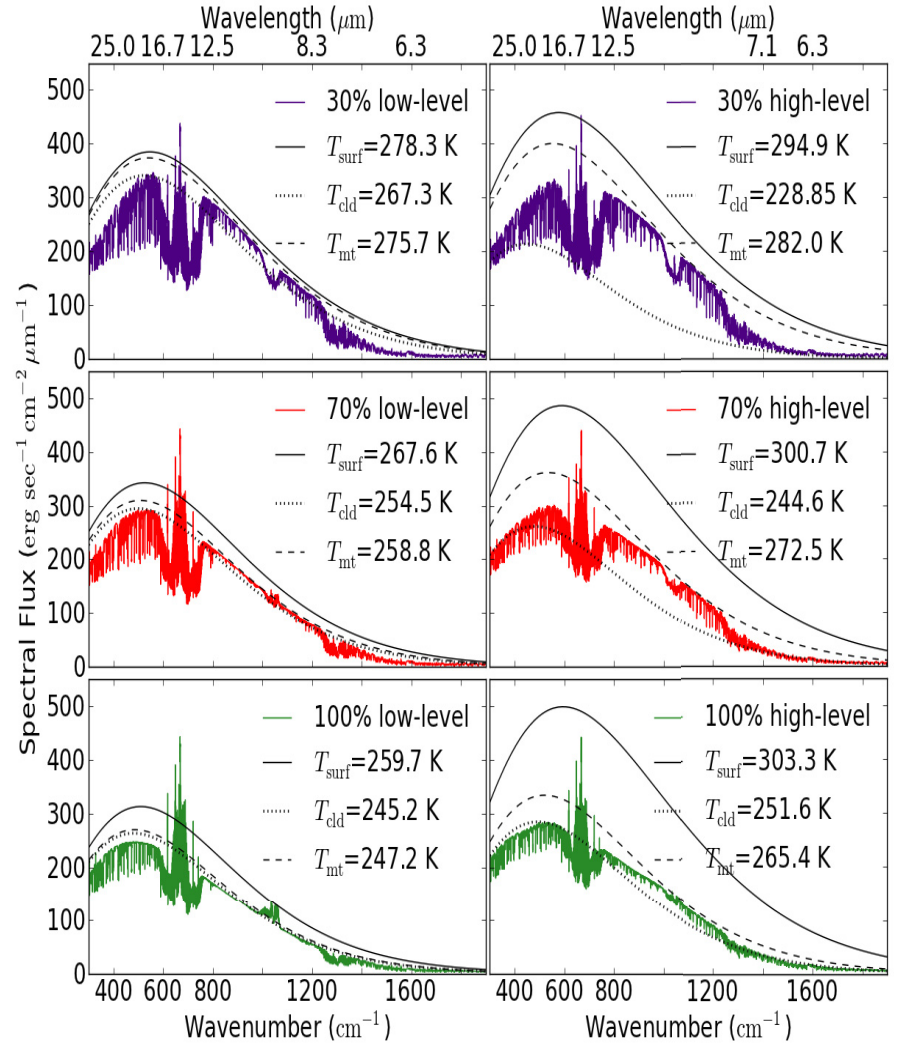

Fig. 5. Spectral flux for the F-star planet under the influence of lowand high-level clouds for different percent coverages. The blackbody curves of the surface temperatures ( $\left.T_{\text {surf }}\right)$, the cloud layer temperatures $\left(T_{\text {cld }},\right)$ and the curves that best match the spectra $\left(T_{\mathrm{mt}}\right)$ are shown for comparison.

effect in the upper stratosphere of the K- and M-star planets than in the other two planets, visible at the center of the band (see Fig. 6-top). In the F- and G-star planets, the low cloud leads to a stronger cooling effect in the troposphere, but in the upper stratosphere, the temperatures are not so affected and are slightly lower compared to the clear atmosphere.

In the band wings (i.e., for wavelengths shorter than $4.2 \mu \mathrm{m}$ and longer than $4.4 \mu \mathrm{m}$ ), the situation is completely different. In these spectral regions, absorption by $\mathrm{CO}_{2}$ is smaller, and radiation coming from lower atmospheric layers reaches the ToA. This absorption occurs above the cloud deck in the case of $100 \%$ low clouds or from levels near the cloud position for partial cover and $100 \%$ high clouds. Since the surface and the tropospheric temperatures are the most affected by the presence of clouds, the impact from the different cloud-cover fraction is higher in these regions.

The high-level cloud impacts the planetary spectra in a different way. In the K- and M-star planets, the center of the $4.3 \mu \mathrm{m}$ band, where radiation arises from the upper atmosphere, is not as affected as when having the low-level cloud, which indicates that the high-level cloud barely affects the stratospheric temperatures of these planets (Fig. 6-bottom). The right wing of the $4.3 \mu \mathrm{m}$ band, between 4.35 and $4.48 \mu \mathrm{m}$, shows higher temperatures with increasing percent coverage, when compared to the clear sky. This occurs because the high-level cloud produces a warming effect on the troposphere of the planets, where the radiation seen at this spectral range mainly originates.

In the F- and G-star planets, the center of the $4.3 \mu \mathrm{m}$ band also shows that the stratospheric temperatures are only slightly 

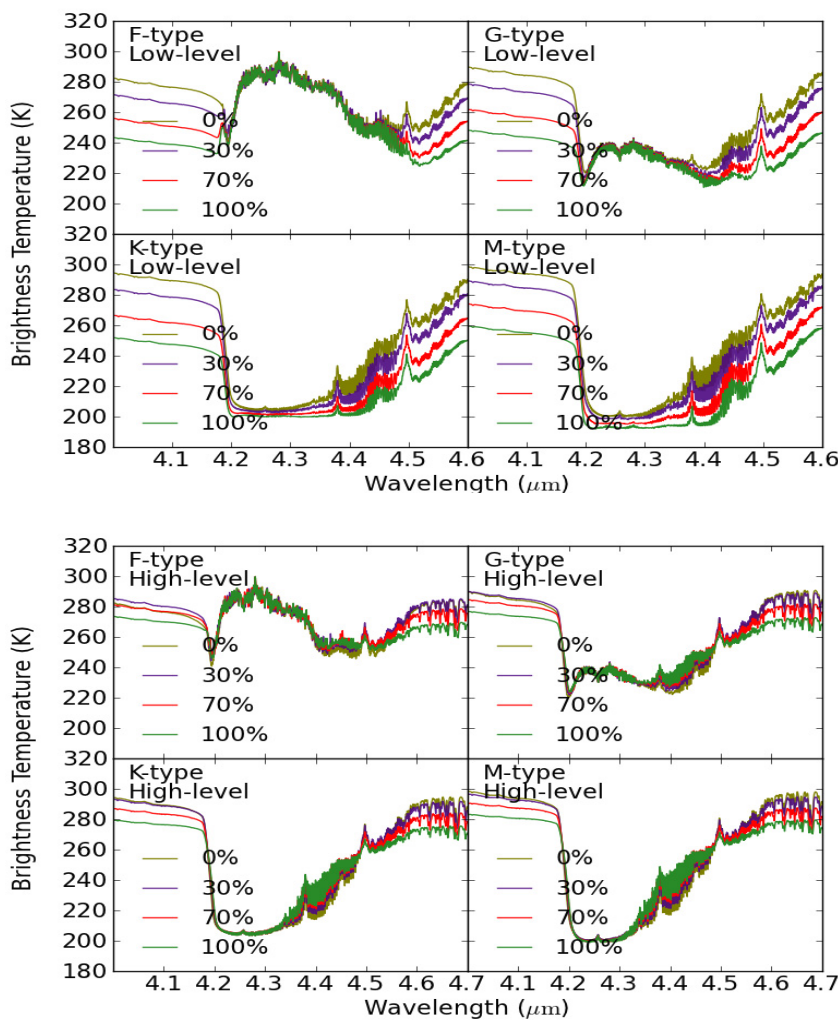

Fig. 6. Spectra in brightness temperature showing the $\mathrm{CO}_{2}$ band at $4.3 \mu \mathrm{m}$ for different planets influenced by low (top) and high (bottom) clouds. The $\mathrm{N}_{2} \mathrm{O}$ band at $4.5 \mu \mathrm{m}$ can also be seen.

affected by the presence of high-level clouds (see also Fig. 6bottom). The effect of high clouds for different coverages at the center of the $4.3 \mu \mathrm{m}$ band is less than in the low cloud cases for all four star-type planets. The explanation is that high clouds affect the stratosphere of the planets to a lesser extent (see Figs. 2 and 4) and this is the region in the atmosphere where the radiation at these wavelengths originates.

Similar effects to those observed at $4.3 \mu \mathrm{m}$ for the different planetary cases can also be seen at the strong $15 \mu \mathrm{m}$ band for low- (Fig. 7-top) and high-level clouds (Fig. 7-bottom). Changes in the state of the upper atmosphere due to the presence of clouds can be observed again at the band center since $\mathrm{CO}_{2}$ presents strong absorption in this region. The $\mathrm{K}$ - and $\mathrm{M}$-star planets are the most affected ones in the stratosphere when influenced by low clouds, while high clouds result in a much lower impact.

\subsubsection{Nitrous oxide}

Nitrous oxide is a uniformly mixed gas found at relatively low concentrations that greatly decrease in the stratosphere. The narrow $\mathrm{N}_{2} \mathrm{O}$ absorption band centered at $4.5 \mu \mathrm{m}$ at the right edge of the $4.3 \mu \mathrm{m} \mathrm{CO}$ band is still distinguishable even for the $100 \%$ cloud cover in all planetary cases (see Fig. 6). In the case of low clouds with total overcast, radiation at ToA mostly arises from regions above the cloud deck (within the troposphere), whereas in the partial coverage cases, radiation receives significant contributions from the tropospheric levels below the cloud as well. The different low cloud coverages have a large impact on tropospheric temperatures, where $\mathrm{N}_{2} \mathrm{O}$ presents more absorption and therefore, the depth of the band decreases, especially for the
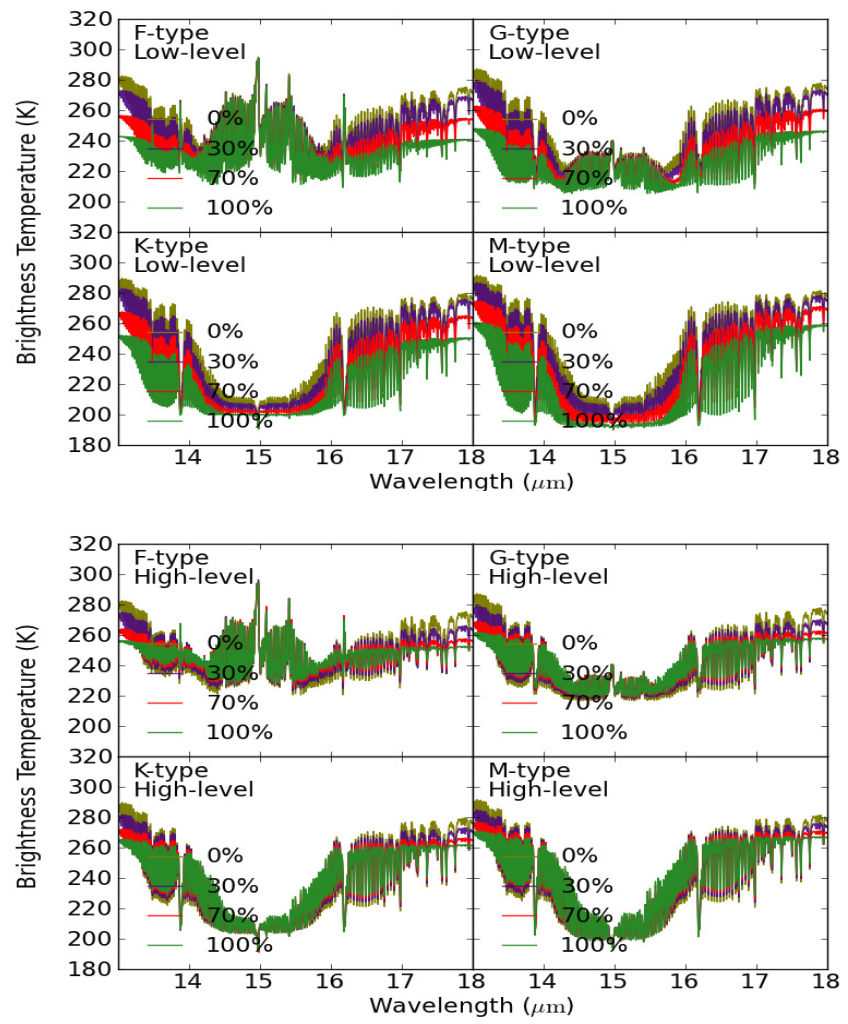

Fig. 7. $\mathrm{CO}_{2}$ band at $15.0 \mu \mathrm{m}$ in the presence of low (top) and high-level (bottom) clouds.

$100 \%$ cloud that is emitting at a much lower temperature than the surface and has a strong blocking effect (see Fig. 4).

In the atmospheres influenced by the high-level cloud with total overcast, the radiation seen at the right wing of the $4.5 \mu \mathrm{m}$ band comes mainly from regions below the cloud, since the high cloud has a small optical depth and does not completely block the radiation emitted at lower levels (see Fig. 4).

\subsubsection{Water vapor}

Water vapor shows high concentrations in the low troposphere that rapidly drop with altitude. In the clear sky atmosphere, the band presents absorption arising from the very low troposphere to the upper regions. In the presence of low clouds, the $6.3 \mu \mathrm{m}$ $\mathrm{H}_{2} \mathrm{O}$ band is highly affected for the $100 \%$ coverage in all the different star planets (see Fig. 8-top). By comparing the brightness temperatures to the atmospheric temperatures from Fig. 2, it can be observed that the spectral regions of strong absorption present radiation arising from levels above the cloud deck till the upper troposphere, whereas the radiation originating below the cloud is mostly blocked and cannot be seen (see Fig. 4).

In the high-level cloud for the $100 \%$ coverage, the band is not so reduced (Fig. 8-bottom). As already pointed out in Sect. 2, this type of cloud has a small optical depth (i.e., it is more transparent), which allows the radiation emitted below the cloud deck to reach the ToA (see Fig. 4). Therefore, the strength of the band is not so affected as in the $100 \%$ low-level cloud.

At the very center of the $6.3 \mu \mathrm{m}$ band, the $\mathrm{H}_{2} \mathrm{O}$ absorption is very weak, and it reveals temperatures corresponding to layers close and below the cloud deck. The high-level cloud 

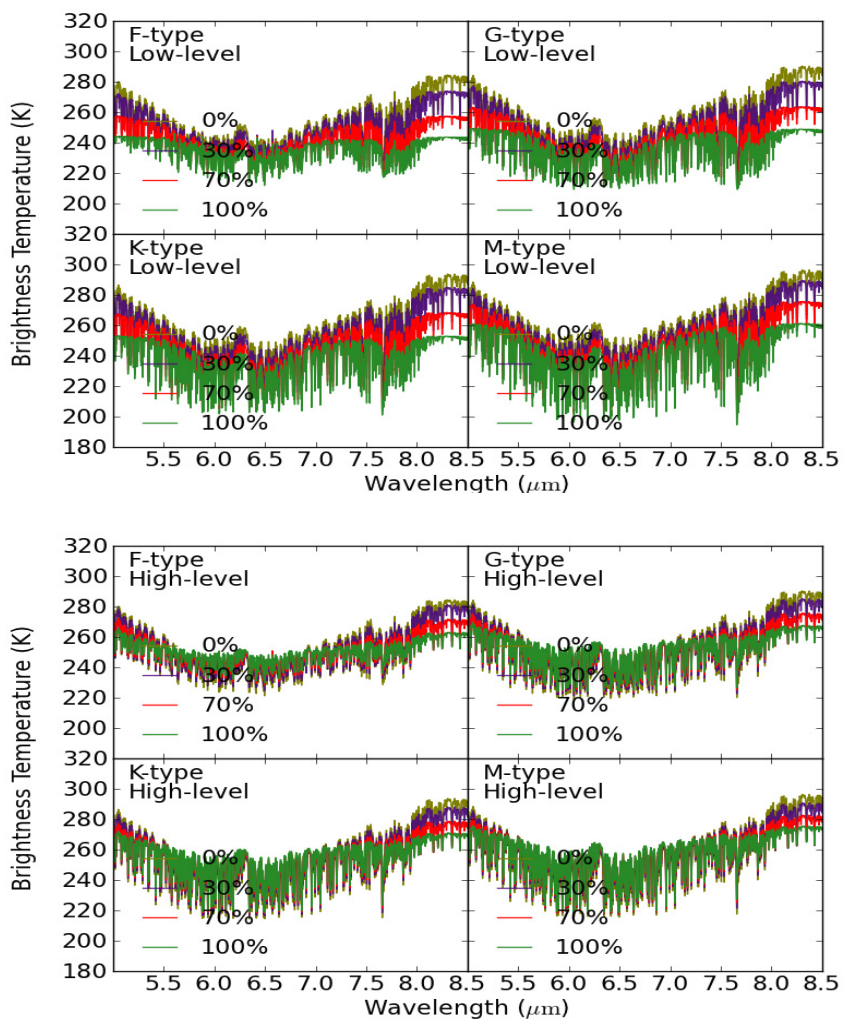

Fig. 8. Spectra showing the $\mathrm{H}_{2} \mathrm{O}$ band at $6.3 \mu$ m influenced by low (top) and high-level (bottom) clouds.

allows radiation originating below the cloud to reach the ToA. Therefore, the differences at the band center between the various high-level cloud coverages and clear spectra are not significant as in the low cloud, where the blocking effect of the layers below is greater and radiation originates at or near the cloud deck.

\subsubsection{Methane}

Methane is a well-mixed gas found in the planet's troposphere with concentrations decreasing in the stratosphere. The $\mathrm{CH}_{4}$ band at $7.7 \mu \mathrm{m}$ is reduced in depth by the different types of clouds and percent coverages. Figure 9 shows that in the cloudfree case, the molecule presents strong absorption in levels with temperatures corresponding to the mid-troposphere (see Fig. 2 and Paper I). Nevertheless, clouds change the tropospheric temperatures and the cloud (100\% coverage in case of low clouds) or nearby (100\% high cloud and partial coverages) layers become the main emitting source, which are all colder than the surface. For both low and high clouds, the spectrum decreases with increasing cloud coverage. Even though the $\mathrm{CH}_{4}$ band shows strong absorption above the cloud deck, the reduction occurs both because the cloud is emitting at a lower temperature than the levels below (where radiation seen at ToA originates from in case of the cloud-free case) and because absorption by $\mathrm{CH}_{4}$ is taking place at layers of temperatures decreased by the cloud. The $\mathrm{CH}_{4}$ band in the F- and G-star planets are the most influenced ones since the atmospheric temperatures of these planets are the most affected ones since the atmospheric temperatures of these planets are more affected than in the K- and M-star planets.

The impact produced by low clouds (see Fig. 9-top) on the $\mathrm{CH}_{4}$ band is greater than for high clouds (Fig. 9-bottom), since
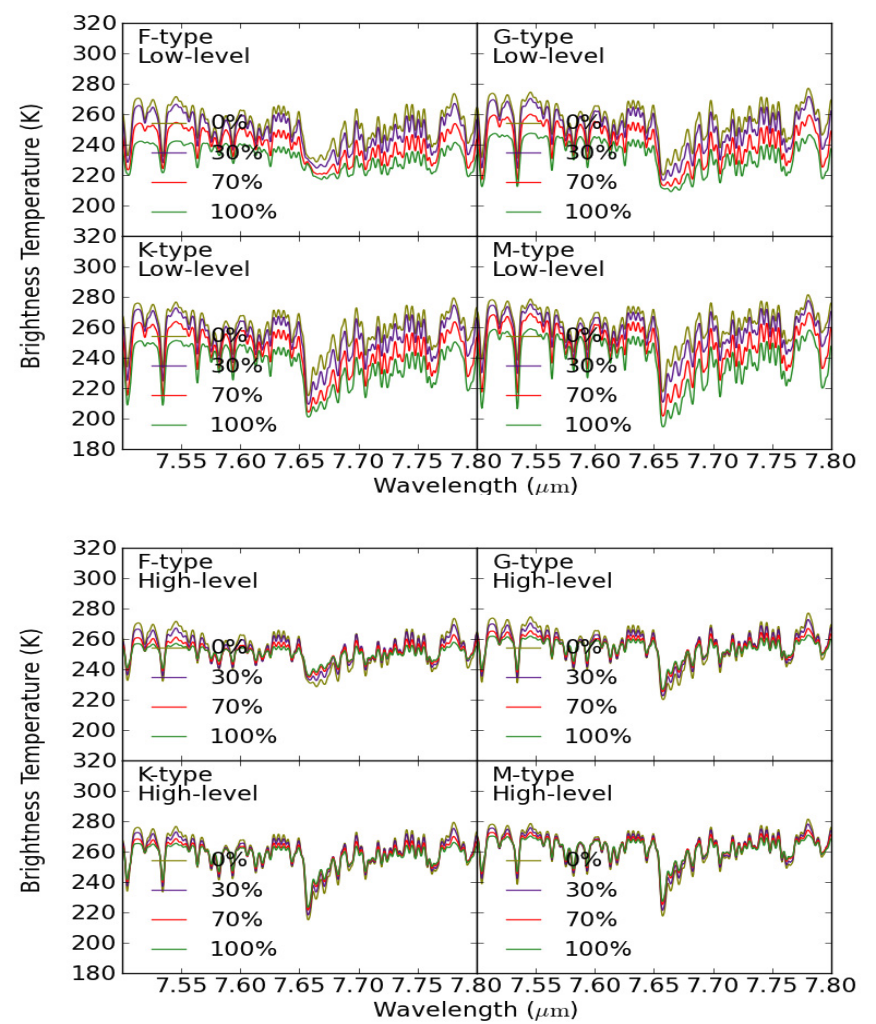

Fig. 9. The $\mathrm{CH}_{4}$ band at $7.7 \mu \mathrm{m}$ in the presence of low (top) and highlevel (bottom) clouds.

they block radiation upwelling from lower layers more efficiently. In the presence of high-level clouds, the tropospheric temperatures (see Fig. 2) are less affected even though they still increase, and the spectrum is not so influenced when compared to the low-level clouds.

\subsubsection{Ozone}

Ozone is found at low concentrations in the troposphere, while its abundance increases in the stratosphere. The very center of the band, where the absorption is relatively weak, indicates temperatures corresponding to the low troposphere for the $0 \%$ cloud cover. In the presence of clouds, the temperatures appearing in the absorption band of the molecule (i.e. roughly $9.4 \mu \mathrm{m}-9.5 \mu \mathrm{m}$ and $9.7 \mu \mathrm{m}-10 \mu \mathrm{m})$ correspond to the mid-stratosphere.

In the presence of low-level clouds, one only sees temperatures corresponding to levels close to the cloud deck, since the flux coming from below the cloud is partially and completely blocked by the $70 \%$ and $100 \%$ coverages, respectively. In the F-star planet, the shape and depth of the $\mathrm{O}_{3}$ band at $9.6 \mu \mathrm{m}$ for the low-level cloud coverages of $70 \%$ and $100 \%$ is strongly affected when compared to the $0 \%$ and $30 \%$ cases (Fig. 10-top). The band takes a different shape for the $100 \%$ low-level cloud, since the main emitting source (cloud deck) is at a lower temperature than the mid-stratosphere for the F-star planet. For the $70 \%$ cloud cover, the temperatures from the mid-stratosphere are very similar to that of the cloud deck, giving the flat appearance to the band. However, in the G-, K-, and M-star planets for all different low-level cloud coverages, the shape of the $\mathrm{O}_{3}$ band remains unchanged, since the mid-stratosphere is at a lower temperature than the levels close to the cloud deck. Moreover, this points out that the F-star planet possesses a much warmer 

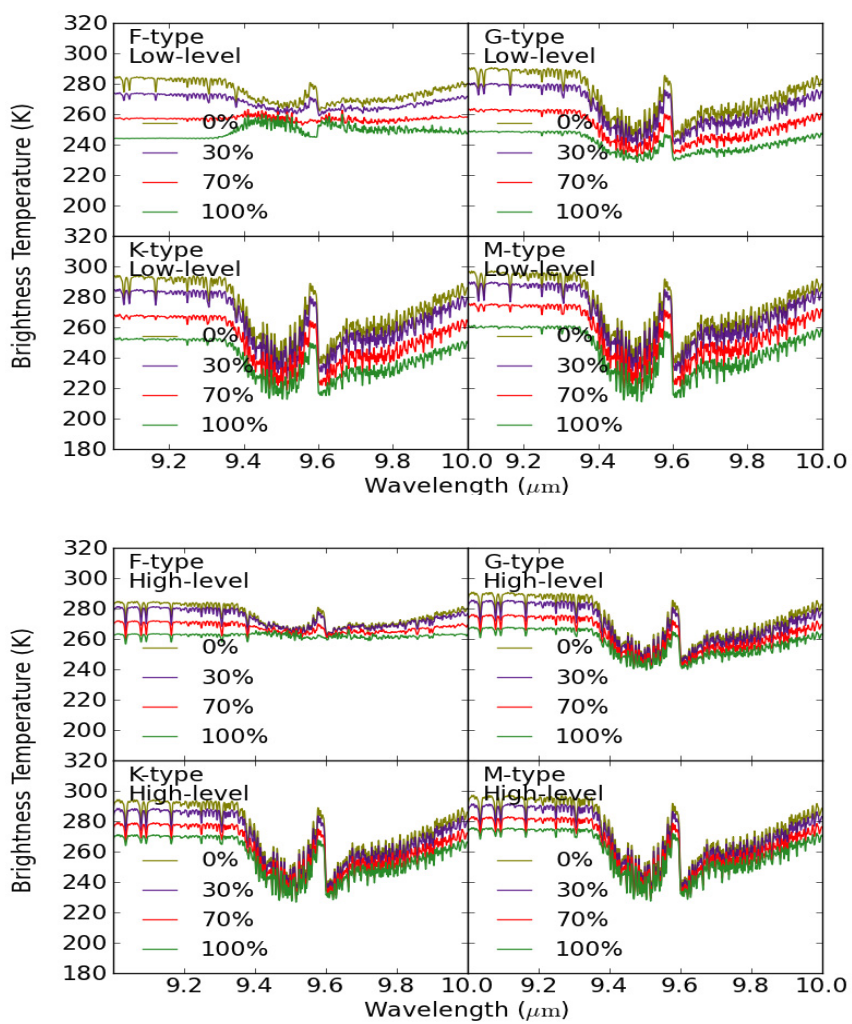

Fig. 10. $\mathrm{O}_{3}$ band at $9.6 \mu \mathrm{m}$ in the presence of low (top) and high-level (bottom) clouds.

mid-stratosphere than the rest of the planets. In the spectrum influenced by the $70 \%$ low-level cloud, the $\mathrm{O}_{3}$ band becomes very flat, which could lead to false negative results.

The $70 \%$ and $100 \%$ high-level cloud coverages have a strong impact as well on the $\mathrm{O}_{3}$ band for the F-star planet (Fig. 10bottom). The altitudes close to the cloud deck where the main energy is being emitted are at temperatures that are comparable to those of the mid-stratosphere, which produces a reduction in the depth of the band. For the rest of the planetary cases, the $\mathrm{O}_{3}$ band is clearly distinguishable even for the total overcast cloud.

The reduction of the depth of the $\mathrm{O}_{3}$ band could lead to the false negative, thus indicating its absence. However, the wrong interpretation of the $\mathrm{O}_{3}$ band could be avoided by studying other spectral regions simultaneously. In particular, the temperature of the effective bottom-level (i.e. cloud or surface essentially) can be inferred in the atmospheric window $(8-12 \mu \mathrm{m})$. Additionally, the 4.3 and $15 \mu \mathrm{m} \mathrm{CO}_{2}$ bands contain information about atmospheric temperatures between the effective bottom and the upper atmosphere. Combining all this information makes it possible to discard $\mathrm{O}_{3}$ false negatives. If the spectrum is flat around $9.6 \mu \mathrm{m}$, and the temperature at the ozone level (estimated from the $\mathrm{CO}_{2}$ bands) differs considerably from the temperature of the effective bottom (estimated from the atmospheric window), then it is very likely that $\mathrm{O}_{3}$ is absent in the atmosphere. This analysis can also be applied for other molecules to discard false negatives.

\subsection{Spectra at different resolutions}

The spectra of planets around F, G, K, and M stars have been simulated at different resolutions under the influence of a $100 \%$ cloud coverage, due to the strong effect that it produces on the

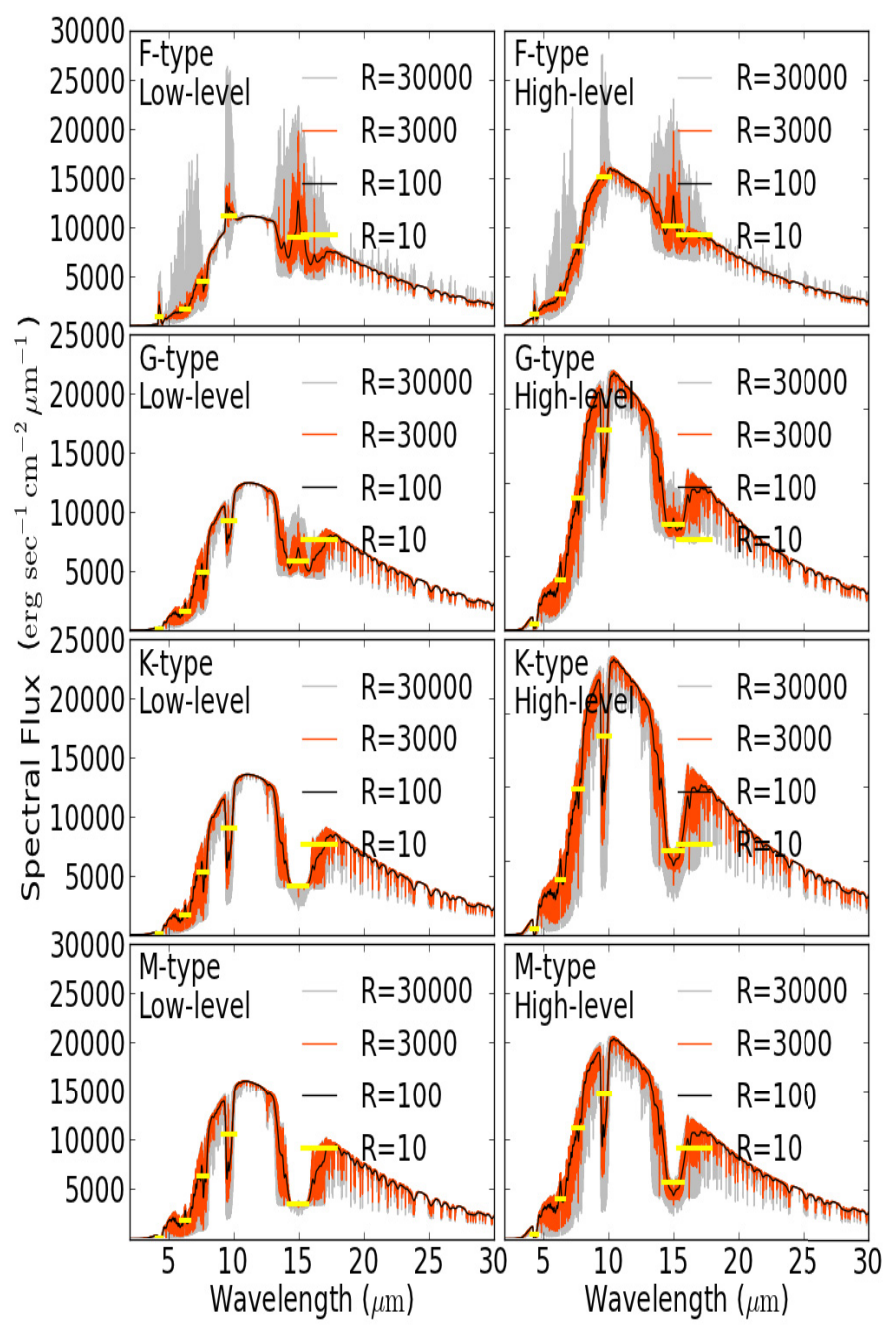

Fig. 11. Spectra of Earth-like planets with a $100 \%$ cloud cover at different resolutions.

spectral molecular bands. Figure 11 shows the spectra at different resolutions under the influence of low and high cloud coverages. It turns out that the most indistinguishable bands result for the spectral resolutions of 10 and 100 in the F-star planet. This planet presents a strong temperature inversion in its stratosphere with temperatures comparable to those found in the troposphere or even higher than the surface temperature that, as a consequence, produces greater effects on the spectral appearance of the different molecules. Moreover, the impact of clouds on the absorption bands is greater, since the contrast between the stratospheric temperatures and the cloud deck (or levels close to the cloud deck) is not as large as for the rest of the planets. Owing to the strong effect that clouds produce on the F-star planet, the study of the spectral resolution presented in this section is focused on this one.

The $\mathrm{CO}_{2}, \mathrm{H}_{2} \mathrm{O}$, and $\mathrm{O}_{3}$ absorption features can still be distinguished at a resolution of 100 in all planetary cases. However, at a spectral resolution of 10 , the $\mathrm{CO}_{2}$ band at $4.3 \mu \mathrm{m}$ cannot be separated from the $\mathrm{N}_{2} \mathrm{O}$ band, as already mentioned in Paper I. To assess the presence of $\mathrm{CO}_{2}$ at $4.3 \mu \mathrm{m}$, the actual spectrum of the F-star planet (Fig. 12) has been compared to the spectrum without $\mathrm{CO}_{2}$. The difference between the two spectra in the central bin is larger compared to that between the side bins. Larger differences can be seen under the influence of the low-level cloud 


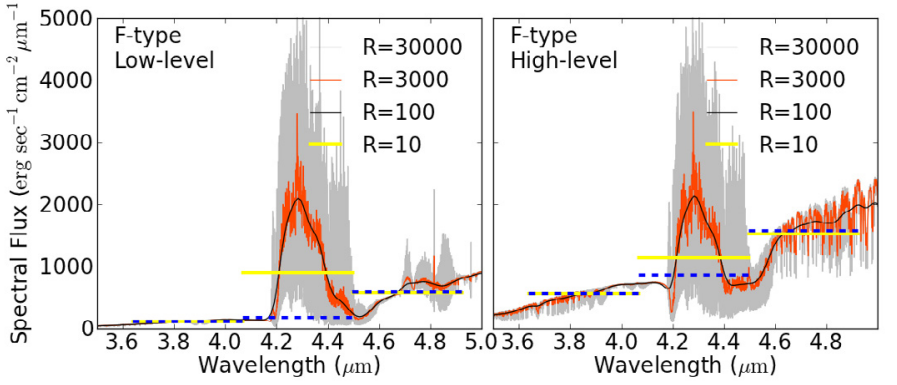

Fig. 12. Spectra showing the $\mathrm{CO}_{2}$ band at $4.3 \mu \mathrm{m}$ in the presence of low (left) and high (right) clouds. An atmosphere without $\mathrm{CO}_{2}$ (blue) is compared to an atmosphere containing all molecules (yellow) at a low spectral resolution of 10 .

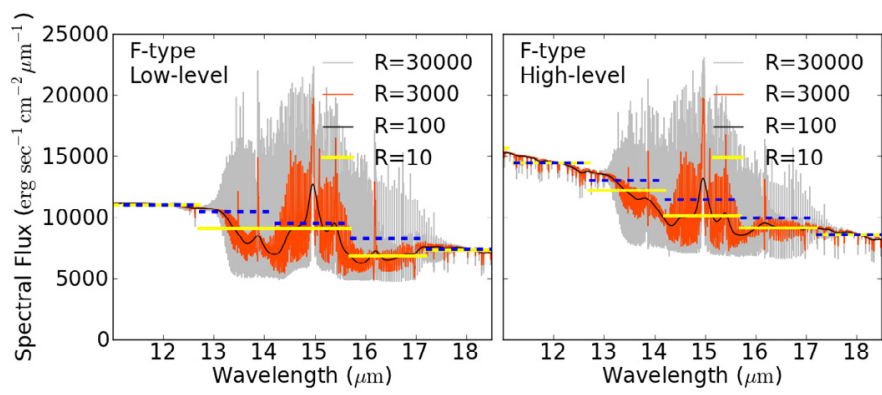

Fig. 13. Spectra showing the $\mathrm{CO}_{2}$ band at $15 \mu \mathrm{m}$ influenced by low (left) and high (right) clouds. An atmosphere in the absence of $\mathrm{CO}_{2}$ (blue) is compared to an atmosphere containing all molecules (yellow) at a low spectral resolution of 10 .

for the drop in the central bin. Consequently, the presence of $\mathrm{CO}_{2}$ can still be determined at $4.3 \mu \mathrm{m}$ in the presence of clouds.

In the $15 \mu \mathrm{m}$ band influenced by the $100 \%$ high-level cloud, the differences between the center bin and the outermost side bins with and without $\mathrm{CO}_{2}$ can also be seen. These differences are smaller in the presence of the low-level cloud. In Fig. 13, it is possible to observe that in the absence of the molecule, the flux in the center bin of the $15 \mu \mathrm{m}$ band increases, but the opposite effect occurs at $4.3 \mu \mathrm{m}$. The $4.3 \mu \mathrm{m}$ band is stronger and therefore, the net effect it produces is emission due to the stratospheric inversion found in the F-star planet. In the $15 \mu \mathrm{m}$ band, the net effect that the molecule produces is absorption, which results in an increase in the spectral flux in the absence of $\mathrm{CO}_{2}$.

The absorption by $\mathrm{H}_{2} \mathrm{O}$ has a contribution from spectral lines and from continuum, which can be found everywhere within the spectral range. Therefore, the presence of $\mathrm{H}_{2} \mathrm{O}$ has an effect on the absolute values of the whole spectrum.

In the previous section, the F-star planet spectra with total overcast showed that the $\mathrm{O}_{3}$ absorption at $9.6 \mu \mathrm{m}$ is less distinguishable than in the presence of partial cloud coverages. This could lead to the wrong conclusion that the molecule is not present in the planetary atmosphere under the $100 \%$ overcast conditions and at a spectral resolution of 10, since the contribution from $\mathrm{O}_{3}$ to the $9.6 \mu \mathrm{m}$ region is highly reduced, and at this mentioned resolution the band is not separable from others. Figure 14 shows the contrast between the $\mathrm{O}_{3}$ band and the neighboring bands. It can be seen that in the presence of the low cloud, the decrease of flux in the center of the $9.6 \mu \mathrm{m}$ region with and without $\mathrm{O}_{3}$ is larger than in the side bins. In the case of the high-level cloud, the drop in flux at the band center is very small so the difficulty of assessing the presence of $\mathrm{O}_{3}$ is greater than in the presence of the low-level cloud.

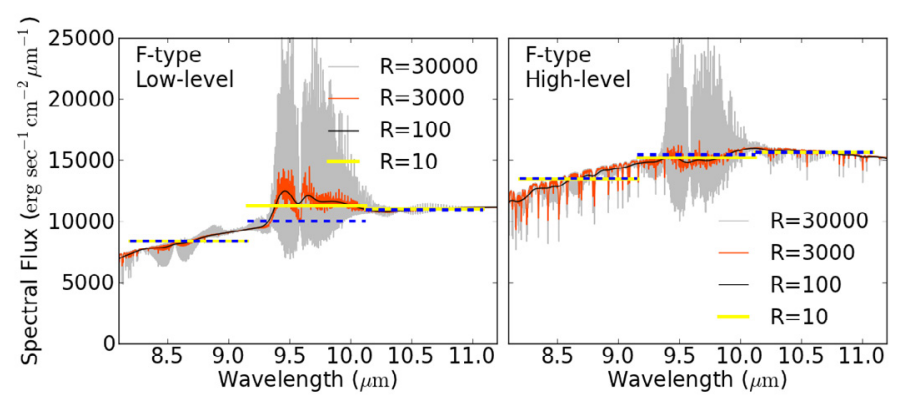

Fig. 14. Spectra showing the $\mathrm{O}_{3}$ band at $9.6 \mu \mathrm{m}$ for a $100 \%$ low (right) and high (left) cloud cover. An atmosphere without $\mathrm{O}_{3}$ (blue) is compared to an atmosphere containing all molecules (yellow) at a low spectral resolution of 10 .

The $\mathrm{CH}_{4}$ band at $7.7 \mu \mathrm{m}$ is distinguishable at a resolution of 100 in both cloud cases. Nevertheless, as stated in Paper I at a resolution of ten the band cannot be separated from the $\mathrm{H}_{2} \mathrm{O}$ band, and the difficulty of establishing the presence of the molecule raises. By following the same technique as used to assess the presence of the dominant molecules of the spectra, it is also possible to determine the presence of $\mathrm{CH}_{4}$. The strength of the $7.7 \mu \mathrm{m} \mathrm{CH} \mathrm{CH}_{4}$ band is also highly affected by the presence of the $100 \%$ low and high cloud coverage, but as one can see in Fig. 15(-left), the differences between the center and the side bins with and without $\mathrm{CH}_{4}$ are not negligible. To establish whether the contributions to the $7.7 \mu \mathrm{m}$ region mainly comes from $\mathrm{CH}_{4}$ or from $\mathrm{H}_{2} \mathrm{O}$, the same approach was performed for the $\mathrm{H}_{2} \mathrm{O}$ molecule (Fig. 15-right).

In the center bin of the spectra influenced by the lowlevel clouds, the $\mathrm{H}_{2} \mathrm{O}$ molecule has an insignificant contribution (Fig. 15-top-right). This is because the low-level cloud has a blocking effect on the levels below the cloud deck, where some of the tropospheric $\mathrm{H}_{2} \mathrm{O}$ emission passes through the cloud and contributes strongly to the $7.7 \mu \mathrm{m}$ region at $R=10$. Consequently, the $\mathrm{CH}_{4}$ molecule is the main contributor to the $7.7 \mu \mathrm{m}$ region, contrary to the cloud-free atmospheric case, where the $\mathrm{H}_{2} \mathrm{O}$ molecule is the main contributor (see Paper I). This facilitates any assessment of the presence of $\mathrm{CH}_{4}$ in the planet.

In the presence of the $100 \%$ high-level cloud, the $\mathrm{H}_{2} \mathrm{O}$ molecule is the main contributor to the $7.7 \mu \mathrm{m}$ region (see Fig. 15-bottom-right). As stated in the previous section, some of the absorption taking place below the cloud deck can be seen under these cloudy conditions. Therefore, this makes $\mathrm{H}_{2} \mathrm{O}$ the main contributor, since the $7.7 \mu \mathrm{m} \mathrm{CH}_{4}$ band cannot be separated from the interfering neighboring $\mathrm{H}_{2} \mathrm{O}$ band at a spectral resolution of 10. However, the contribution by $\mathrm{CH}_{4}$ to the $7.7 \mu \mathrm{m}$ region can still be assessed.

In the top left of Fig. 16, the contribution by $\mathrm{N}_{2} \mathrm{O}$ at $4.5 \mu \mathrm{m}$ for the low-level cloud is negligible, and the main contributor at this wavelength is $\mathrm{CO}_{2}$. For the high-level cloud, $\mathrm{N}_{2} \mathrm{O}$ makes a greater contribution to the band; nevertheless, the $\mathrm{CO}_{2}$ molecule is still the main absorber (Fig. 16-bottom-right).

\subsection{Comparison to low-resolution studies}

Since the atmospheric profiles and cloud optical properties used to calculate the spectra of Earth-like planets in the present paper have already been used by Kitzmann et al. (2011, herein called Kitzmann11 in this section) to produce low-resolution spectra, 
M. Vasquez et al.: IR radiative transfer in atmospheres of Earth-like planets influenced by clouds

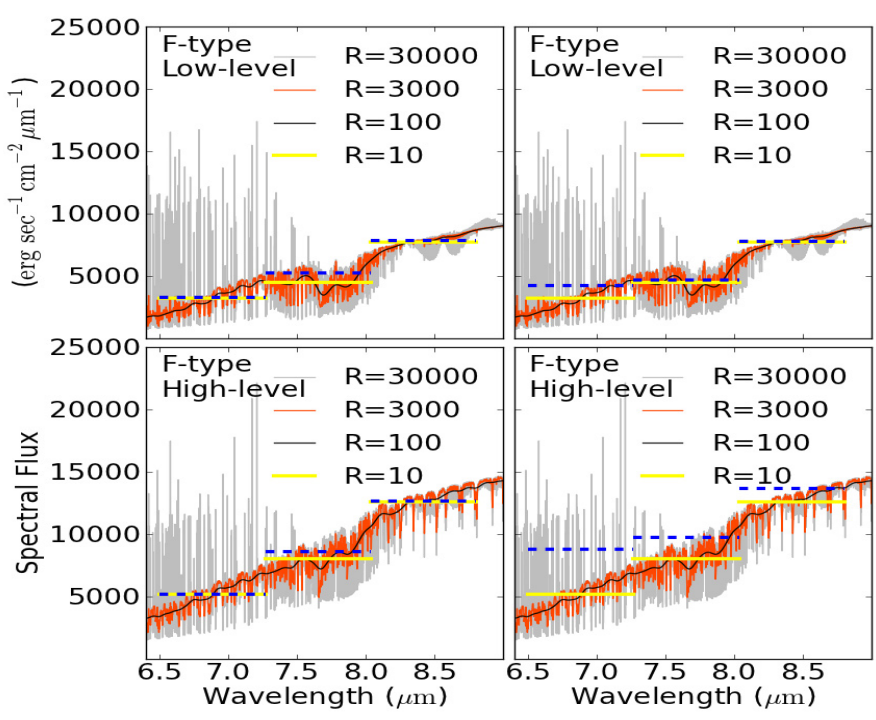

Fig. 15. Spectra showing the $\mathrm{CH}_{4}$ band at $7.7 \mu \mathrm{m}$ for $100 \%$ low (top) and high-level (bottom) cloud coverages. The figure on the left shows an atmosphere with all molecules (yellow) and without $\mathrm{CH}_{4}$ (blue) and the one on the right, an atmosphere with and without $\mathrm{H}_{2} \mathrm{O}$ at a resolution of 10 .

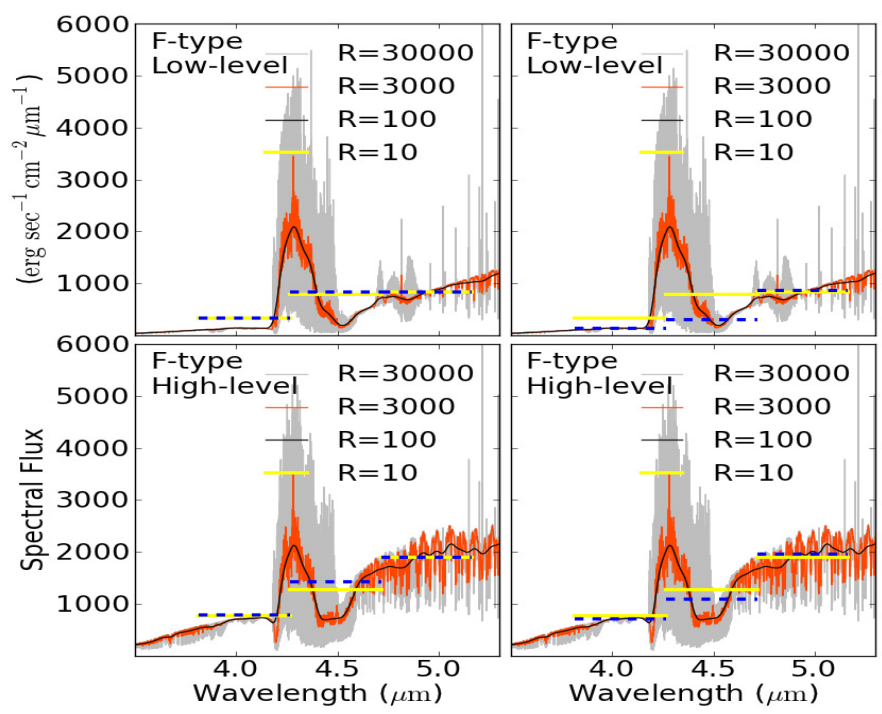

Fig. 16. Spectra showing the $\mathrm{N}_{2} \mathrm{O}$ band at $4.5 \mu \mathrm{m}$ influenced by $100 \%$ low (top) and high-level (bottom) cloud coverages. The figure on the left shows an atmosphere with (yellow) and without (blue) the presence of $\mathrm{N}_{2} \mathrm{O}$ and the one on the right, an atmosphere with and without $\mathrm{CO}_{2}$ for resolution 10 .

both resulting computations at resolutions $1<R<40$ are compared in this section.

Figure 17 illustrates the spectra modeled with GARLIC and RRTM/2s from Kitzmann11 for the four different planets with the $0 \%$ cloud coverage and for the $100 \%$ low- and high-level clouds. It can be seen that, in general, the spectra calculated using GARLIC shows slightly lower fluxes than the spectra calculated using RRTM/2s.

The IR radiative transfer model (RRTM/2s) in Kitzmann11 consists of a hemispheric mean two-stream method (Toon et al. 1989), while the gaseous absorption is described by the correlated k-method via the RRTM model (Mlawer et al. 1997). GARLIC considers line-by-line and discrete ordinate methods. For all the calculations presented in this paper, a four-stream

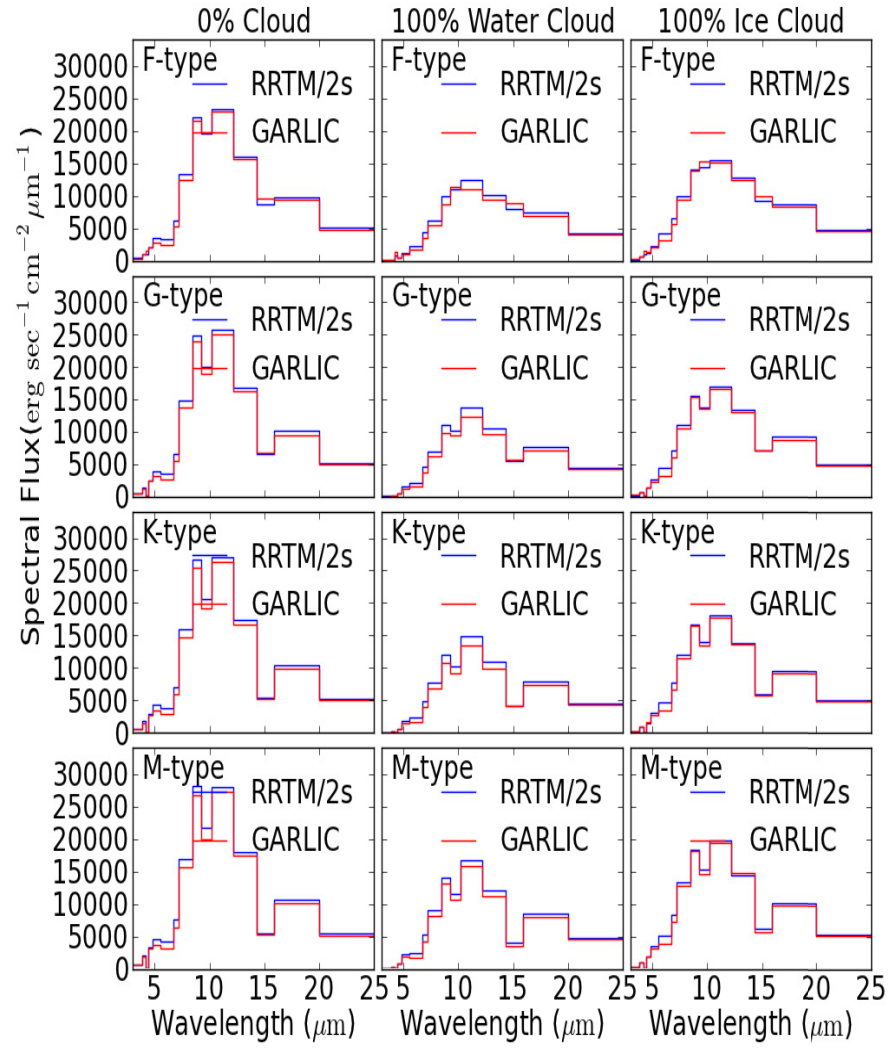

Fig. 17. Low- resolution spectra $(1<R<40)$ calculated with GARLIC and RRTM/2s are shown for comparison.

approach was selected. To test the sensitivity of the spectrum to different numbers of streams, computations using GARLIC and the two-stream radiative transfer model, RTSPEC (Deeter \& Evans 1998), were performed. Also, the sensitivity of the spectrum to different spectroscopy databases were tested (RRTM $\mathrm{k}$-distribution is based on HITRAN 96). However, in none of these studies does the spectra show a significant difference, which leads to the conclusion that the main differences arise from the different methods that were used to account for the spectral dependency of the atmospheric properties (correlated-k and line-by-line treatments).

Due to the limitations of the tabulated k-distribution data, Kitzmann11 did not investigate the impact of the various absorbers on the spectra by excluding a particular molecule. Therefore, computations of the spectra using the same procedure as in Sect. 3.2, with and without the selected molecules, have been carried out for the low-resolution spectra $(1<R<40)$ to determine whether the main absorbing molecules are identifiable. For this, only the planet around the F star has been considered, since based on the previous results, this one presents a large temperature inversion in the upper atmosphere that has a strong impact on the spectra. The $100 \%$ cloud cover has been used in the comparisons due to the extremity of the case, as well as the $0 \%$ cloud cover, which was analyzed in Paper I. The spectral bands examined in the previous sections of this work have been considered in this study to determine the main molecular contributor to each one of them.

In Fig. 18-top, the spectra influenced by the $100 \%$ low-level cloud are shown with and without the main absorbing molecules in the thermal region. At $9.6 \mu \mathrm{m}$ the main contributor is $\mathrm{O}_{3}$, as can be seen from the large difference that the spectrum without the molecule presents when compared to the one containing all 


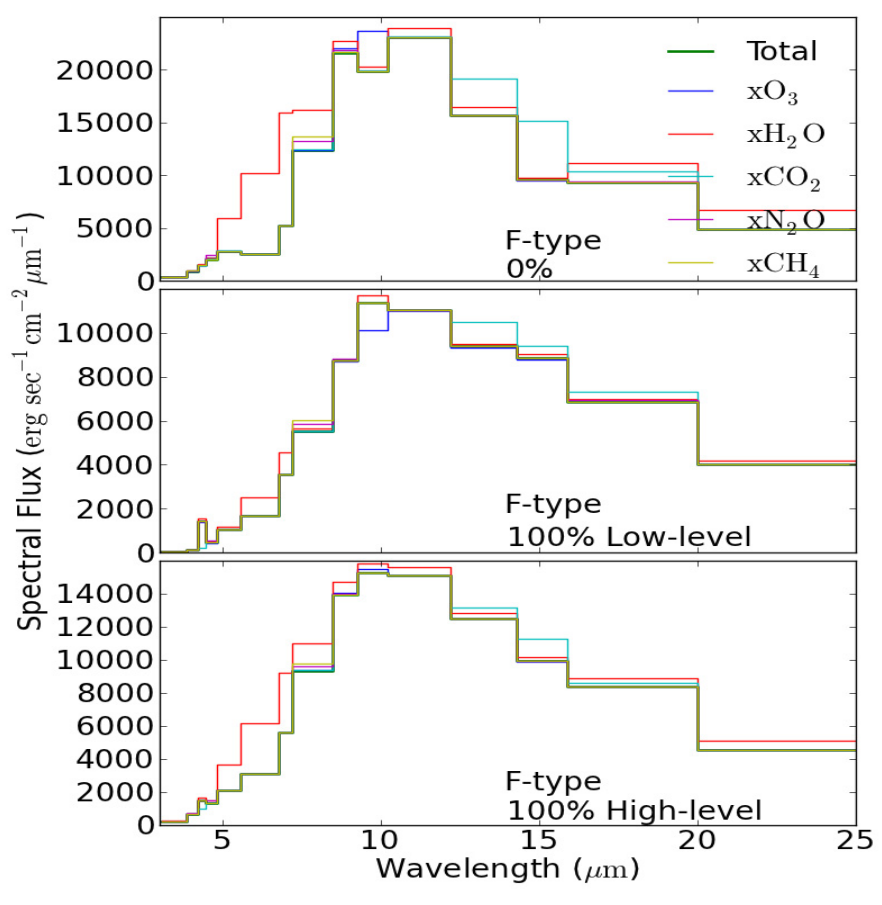

Fig. 18. Low-resolution spectra $(1<R<40)$ calculated using GARLIC with all main molecules (total) and in the absence of them. The spectra are shown in the absence of clouds (top) and in the presence of low(middle) and high-level (bottom) clouds.

molecules. This is also the case for the cloud-free case (see also Fig. 18-mid). However, in the presence of the high-level cloud, the molecule having the greatest impact on the $9.6 \mu \mathrm{m}$ region is $\mathrm{H}_{2} \mathrm{O}$ (see Fig. 18-bottom).

In the cloud-free spectrum at $R=10$, the $\mathrm{H}_{2} \mathrm{O}$ molecule makes a greater contribution to the $4.3 \mu \mathrm{m}$ region; however, in the presence of clouds the $\mathrm{CO}_{2}$ becomes the main contributor. As shown in Sect. 3.1, the low- and high-level clouds partially block the levels below the cloud deck, where $\mathrm{H}_{2} \mathrm{O}$ is absorbing strongly, but for the $0 \%$ cloud cover, the absorption taking place at these low atmospheric levels reaches the ToA. Therefore, for the cloud-free case, the $\mathrm{CO}_{2}$ molecule cannot be identified as the main contributor at $4.3 \mu \mathrm{m}$. In the $15 \mu \mathrm{m}$ region, $\mathrm{CO}_{2}$ is the major molecule characterizing the band in the presence of clouds and in the absence of them.

The $7.7 \mu \mathrm{m} \mathrm{CH}_{4}$ band is highly affected by $\mathrm{H}_{2} \mathrm{O}$ absorption, which has even a greater impact than $\mathrm{CH}_{4}$ for the cloud-free and high-level cloud. Nevertheless, for the low-level cloud, $\mathrm{CH}_{4}$ is still the leading absorbing molecule. It was already shown in Sect. 3.1 (Fig. 4) that even though the water cloud is located at a lower altitude than the ice cloud, it strongly attenuates the radiation arising from the levels where $\mathrm{H}_{2} \mathrm{O}$ presents strong absorption. This fact makes the $\mathrm{CH}_{4}$ molecule dominate at $7.7 \mu \mathrm{m}$ in the presence of the low-level cloud.

$\mathrm{N}_{2} \mathrm{O}$ dominates the region at $4.6 \mu \mathrm{m}$ for the $0 \%$ and $100 \%$ low-level cloud coverages. For the high-level cloud, the levels where $\mathrm{N}_{2} \mathrm{O}$ presents stronger absorption are partially blocked, and the radiation that reaches the To $\mathrm{A}$ at these wavelengths is mostly due to absorption by $\mathrm{H}_{2} \mathrm{O}$.

Kitzmann11 stated that for high-percent cloud coverages, the $\mathrm{O}_{3}$ and $\mathrm{CO}_{2}$ absorption features were not distinguishable in the planet of the $\mathrm{F}$ star. However, based on the more detailed analysis presented above, one can state that $\mathrm{CO}_{2}$ is still identifiable, even for the $100 \%$ cloud cover at 4.3 and $15 \mu \mathrm{m}$. On the other hand,
$\mathrm{O}_{3}$ is distinguishable at $9.6 \mu \mathrm{m}$ for the $100 \%$ low cloud, but not in case of the $100 \%$ high cloud.

\section{Discussion}

This study is confined to cloud-covered Earth-like planets orbiting main sequence stars. Clouds in other objects outside the solar system have already been extensively studied. Brown dwarfs are very massive and very different from terrestrial planets in terms of, say, their atmospheric structure, pressure, chemical composition, and scattering matter properties. Nevertheless, the wide variety of cloud properties encountered serves as a guide for exploring other planetary bodies, such as hot Jupiters. Therefore, the study of clouds in substellar atmospheres gives an idea about the wide variety of species that can potentially condense in planetary atmospheres and their effects on e.g. the spectral appearance. This provides a wide range of scenarios that might help for understanding the radiative transfer in the cloudy atmospheres of terrestrial exoplanets.

The planet around the G-star could be considered as an Earth twin, whereas similarity to Earth conditions for F-, K-, and M-star planets could only be achieved by scaling the distance in order to assure similar incoming "solar" energy, thereby achieving moderate Earth-like surface conditions. However, upper atmospheric conditions are significantly different from Earth, such as for the lack of an inversion for the M-star planet or the high stratopause temperatures for the F-star planet. This restriction to Earth-like planets is in line with most papers investigating the spectral appearance of exoplanets and the detectability of biosignatures (see Sect. 1). Clearly it would be interesting to study radiative transfer for a much larger class of exoplanets. However, the actual state of numerous exoplanets discovered in recent years is likely to be way beyond our imagination, and defining atmospheric scenarios (using coupled radiation convection - chemistry models) required as input for radiative transfer modeling could easily become speculation.

The diversity of possible gas atmospheres is already large, but clouds clearly add another dimension of complexity. To focus on the cloud impact on biosignatures, we limit our study to water and ice clouds as found on Earth. In particular we excluded, for example, $\mathrm{H}_{2} \mathrm{SO}_{4}$ or $\mathrm{CO}_{2}$ clouds known from other planets (e.g. Venus and present Mars) because in these hostile environments, the probability of life (as we know it) is drastically reduced. In view of the wide diversity of clouds we further confined our study to two important, yet rather distinct, cloud types (see Kitzmann et al. 2010 for a discussion of this choice).

Different cloud parameters make an impact on the radiative transfer. Particle type and size distribution influence the cloud optical properties, hence the radiative forcing and ultimately the atmospheric conditions. Particles of larger optical depth have a greater impact on radiative transfer and, accordingly, on the strength and shape of the molecular absorption bands. This may also raise difficulties when determining and identifying the molecules that contribute the most at the different spectral bands, especially at low resolution.

The cloud optical properties vary with particle size and composition. Typically, larger cloud particles lead to larger optical depths and sharper forward-peaked phase functions. The refractive index of liquid water and ice water particles has different spectral dependencies leading to different optical properties. Figure 19 shows the cloud optical depth of water and ice cloud particles for two different effective radii, $1 \mu \mathrm{m}$ and $10 \mu \mathrm{m}$, respectively. Although the composition has an impact on the optical depth, the influence of the effective radii is stronger. 
M. Vasquez et al.: IR radiative transfer in atmospheres of Earth-like planets influenced by clouds

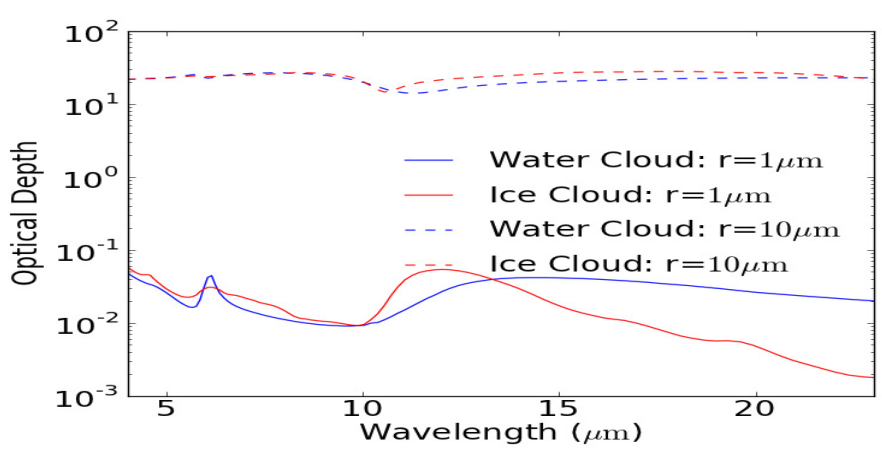

Fig. 19. Optical depths for water and ice cloud particles of effective radius $1 \mu \mathrm{m}$ and $10 \mu \mathrm{m}$.

The weighting functions depend on the cloud optical depth, and implicitly, on the cloud particle size and composition. Since the impact of composition on the optical depth is moderate, the impact on the weighting functions is also moderate. In contrast, the effect of the cloud particle size, through the modification of the optical depth, is large. Figure 20 presents the weighting functions for the F-star planet with 100\% low-level water clouds of effective radii $1 \mu \mathrm{m}$ and $10 \mu \mathrm{m}$. Optically thin clouds have a low impact on the radiative transfer since the amount of photons subjected to extinction events by particles is low. Accordingly, the optically thin $r_{\text {eff }}=1 \mu \mathrm{m}$ cloud allows a considerable amount of photons arising from the atmospheric layers below the cloud deck to reach the ToA. On the other hand, the optically thick $r_{\text {eff }}=10 \mu \mathrm{m}$ cloud blocks most of the photons from subcloud layers and prevents them from reaching the ToA.

Cloud properties affect the radiative transfer, but the impact becomes more or less pronounced depending on the spectral resolution. For optically thin clouds, contributions from molecular emission below and above the cloud reach the ToA. At low spectral resolution, these contributions are convolved. Therefore, optically thin clouds raise difficulties for stratospheric target species (i.e. $\mathrm{O}_{3}$ ). At high spectral resolution, the spectra are highly affected as well, but the mixing of the cloud contributions above and below is less since the instrument can resolve narrower spectral regions. For optically thick cloudy conditions, contributions from below the cloud are mostly blocked. Therefore, the differences at low and high resolution spectra arise from the convolution of contributions above the cloud.

The radiative-convective scheme accounted for the influence of clouds on temperature, pressure, and $\mathrm{H}_{2} \mathrm{O}$ concentrations, whereas an enhanced model would also consider the chemical impact on the abundances of other molecular constituents. Atmospheric clouds influence UV and temperature-profiles, which would give feedback on atmospheric photochemistry via, e.g., photolytic reaction rates and temperature-dependent gasphase reactions. In turn, the photochemistry would influence the atmospheric abundances of radiative gases, such as $\mathrm{CH}_{4}$ and $\mathrm{N}_{2} \mathrm{O}$, which affect the climate. However, since cloud microphysical processes (needed for cloud-chemistry feedback) are timedependent, coupling such processes into a one-dimensional stationary model (Grenfell et al. 2007) is problematic and should be reserved for future work with time-dependent schemes.

Our study can be extended further in several ways: radiative transfer modeling the impact of other cloud compositions and multilayered clouds is possible without any code upgrades. Likewise, analysis of transmission spectra or the short-wave IR is planned. Furthermore, for an assessment of
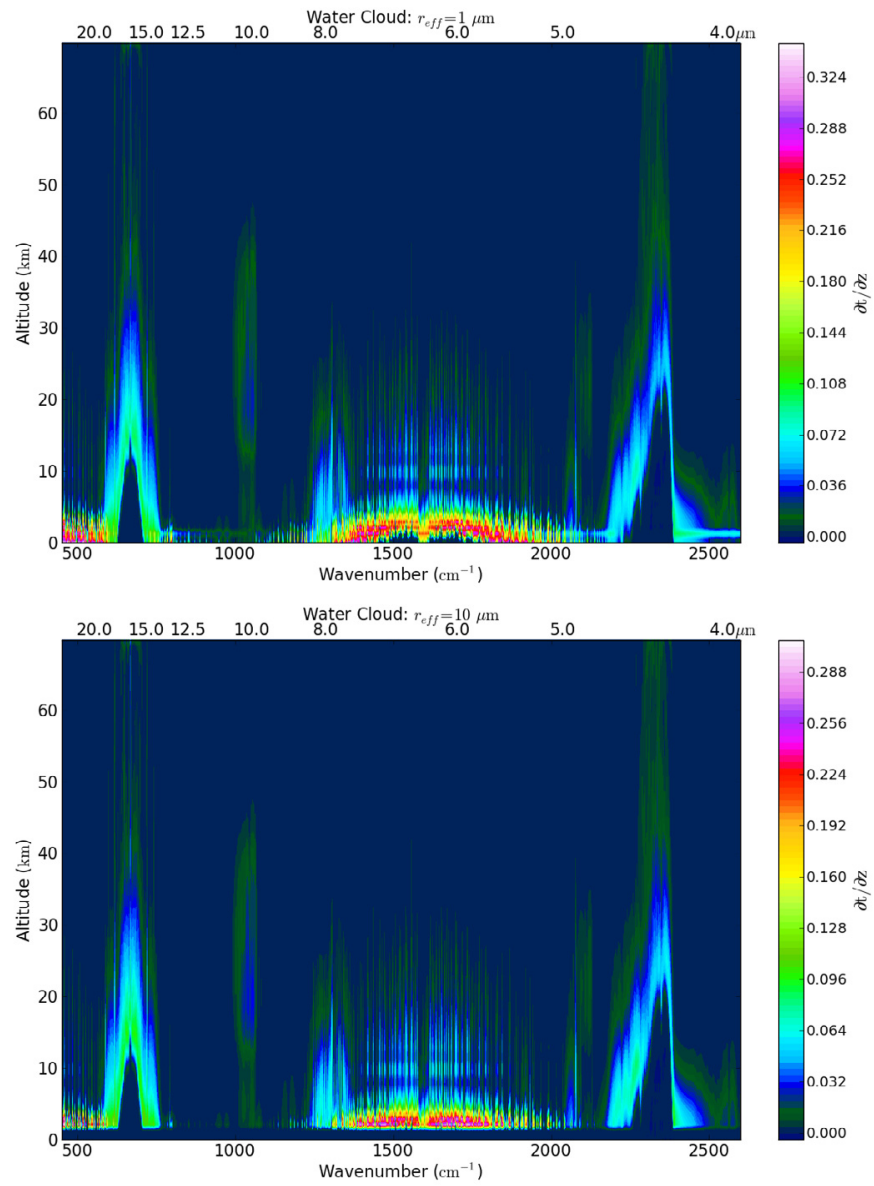

Fig. 20. Weighting functions for the F-star planet influenced by $100 \%$ low-level water clouds of particle radius of $1 \mu \mathrm{m}$ and $10 \mu \mathrm{m}$ at a spectral resolution of 3000 .

future observations, specifications of a more realistic instrument (in particular noise) should be considered.

\section{Summary}

The thermal emission spectra at high and low resolutions of Earth-like planets around different type of stars (F, G, K, and $\mathrm{M}$ stars) were simulated under the influence of single layer low or high-level clouds for different coverages. In general, the different cloud conditions decreased the depth of the molecular band's flux (contrast center vs. wing) and impacted the shape of the high-resolution spectra. Moreover, the strengths of the molecular bands were reduced.

In agreement with Kitzmann et al. (2011) the planet around the $\mathrm{F}$ star was the most affected one. The $\mathrm{H}_{2} \mathrm{O}$ and $\mathrm{O}_{3}$ bands showed a great reduction in depth for total overcast cases and even appeared very flat in shape. In the K- and M-star planets, the effect on the different molecular bands was less than in the planets orbiting the $\mathrm{F}$ and $\mathrm{G}$ stars. The strong $\mathrm{CO}_{2}$ bands at 4.3 and $15 \mu \mathrm{m}$ in all the different planets offered valuable information about stratospheric temperatures, independently of the presence of high- and low-level clouds, since $\mathrm{CO}_{2}$ absorbs throughout the whole atmosphere, as indicated by the weighting functions. This means that the bands are the least affected ones in the thermal region. The $\mathrm{N}_{2} \mathrm{O}$ band at $4.5 \mu \mathrm{m}$ was still distinguishable even for the total overcast in all four different planets.

In the atmospheric window $(8-12 \mu \mathrm{m})$, clouds can prevent any estimation of the surface temperature, which is consistent 
with Kitzmann et al. (2011). In case of the high-level cloud, the temperature difference between the cloud layer and the surface is greater. Even low cloud coverages bias the surface temperature estimation considerably. On the other hand, one can still obtain a good estimate for partially covered planets.

The contributions to different spectral bands were studied in order to determine the main biosignatures in the atmosphere at a spectral resolution of 10 . It was found that the contribution by $\mathrm{CH}_{4}$ to the $7.7 \mu \mathrm{m}$ region can be better established in the presence of the low-level cloud because the $\mathrm{H}_{2} \mathrm{O}$ contribution at this wavelength gets blocked by the cloud. In the high-level cloud, the contribution by $\mathrm{H}_{2} \mathrm{O}$ is greater than the $\mathrm{CH}_{4}$ contribution, which raises the difficulty of identifying the molecule at $7.7 \mu \mathrm{m}$. Contributions of $\mathrm{N}_{2} \mathrm{O}$ at $4.5 \mu \mathrm{m}$ cannot be assessed for the $100 \%$ low-level cloud. In the highly affected $\mathrm{H}_{2} \mathrm{O}$, and $\mathrm{O}_{3}$ bands one can still established their contributions in the planet's atmosphere at a spectral resolution of 10 .

Analysis of individual IR bands of cloud covered planets can lead to false negative interpretations. Nevertheless, these negatives could be avoided by analyzing a combination of certain regions of the spectra (i.e., the atmospheric window, $\mathrm{CO}_{2}$ bands and the desired molecular band). In particular, knowledge of the temperatures is crucial for avoiding false interpretations.

Even for low-resolution spectra, the detailed analysis allowed the different molecules to be identified. Although some information is contained in a low-resolution spectrum, certain information may be missing without any additional study. For some of the molecules (i.e., $\mathrm{CH}_{4}$ and $\mathrm{O}_{3}$ ), the clouds were found to be advantageous to determine their presence.

In conclusion, we used a high-resolution line-by-line multiple scattering radiative transfer model to analyze the IR thermal emission spectra of cloud-covered exoplanets. To our knowledge, Kitzmann et al. (2011) and the present work are the first studies of the radiative transfer of Earth-like planets that use thermodynamically consistent atmospheric scenarios from a coupled radiative-convective climate model including an explicit cloud parameterization. In particular, here we have studied the main IR molecular bands under different cloudy conditions and provided weighting functions to identify the radiation sources. This helps in interpreting the spectral appearance of terrestrial exoplanets, which is essential for retrieving of atmospheric and surface parameters and for seeking habitable worlds.

Acknowledgements. Many thanks to the Helmholtz Research Alliance Planetary Evolution and Life for its financial support. Furthermore, we would like to thank Lee Grenfell for helpful discussions.

\section{References}

Ackerman, A. S., \& Marley, M. S. 2001, ApJ, 556, 872

Barclay, T., Rowe, J. F., Lissauer, J. J., et al. 2013, Nature, 494, 452

Batalha, N. M., Rowe, J. F., Bryson, S. T., et al. 2013, APJS, 204, 24

Bean, J. L., Kempton, E. M.-R., \& Homeier, D. 2010, Nature, 468, 669
Berta, Z. K., Charbonneau, D., Désert, J.-M., et al. 2012, ApJ, 747, 35

Bohren, C., \& Huffman, D. 1983, Absorption and scattering of light by small particles (Wiley)

Borucki, W. J., Koch, D. G., Basri, G., et al. 2011, APJ, 736, 19

Burrows, A., Heng, K., \& Nampaisarn, T. 2011, ApJ, 736, 47

Charbonneau, D., Brown, T. M., Noyes, R. W., \& Gilliland, R. L. 2002, ApJ, 568,377

Clough, S., Kneizys, F., \& Davies, R. 1989, Atmos. Res., 23, 229

Cushing, M. C., Marley, M. S., Saumon, D., et al. 2008, ApJ, 678, 1372

De Kok, R., Stam, D., \& Karalidi, T. 2011, ApJ, 741, 59

Deeter, M., \& Evans, K. F. 1998, J. Quant. Spectr. Radiat. Transf., 60, 635

Des Marais, D. J., Harwit, M. O., Jucks, K. W., et al. 2002, Astrobiology, 2, 153

Fressin, F., Torres, G., Rowe, J. F., et al. 2011, Nature, 482, 195

Gilliland, R. L., Marcy, G. W., Rowe, J. F., et al. 2013, ApJ, 766, 40

Grenfell, J. L., Stracke, B., von Paris, P., et al. 2007, Planet. Space Sci., 55, 661

Grenfell, J., Gebauer, S., von Paris, P., et al. 2011, Icarus, 211, 81

Hanel, R., Conrath, B., Jennigs, D., \& Samuelson, R. 2003, Exploration of the Solar System by Infrared Remote Sensing, 2nd edn. (Cambridge University Press)

Haus, R., \& Arnold, G. 2010, Planet. Space Sci., 58, 1578

Hearty, T., Song, I., Kim, S., \& Tinetti, G. 2009, APJ, 693, 1763

Helling, C., Woitke, P., \& Thi, W.-F. 2008, A\&A, 485, 547

Heymsfield, A., \& Platt, C. 1984, J. Atmos. Sci., 41, 846

Huitson, C. M., Sing, D. K., Vidal-Madjar, A., et al. 2012, MNRAS, 422, 2477

Kaltenegger, L., Traub, W. A., \& Jucks, K. W. 2007, APJ, 658, 598

Kasting, J. F., Pollack, J. B., \& Ackerman, T. P. 1984, Icarus, 57, 335

Kitzmann, D., Patzer, A., von Paris, P., et al. 2010, A\&A, 511, A66

Kitzmann, D., Patzer, A., von Paris, P., Godolt, M., \& Rauer, H. 2011, A\&A, 531, A62

Koch, W. J. B. D. G., Batalha, N., Bryson, S. T., et al. 2012, APJ, 745, 120

Kokhanovsky, A. A. 2006, Cloud Optics (Dordrecht: Springer)

Lovis, C., Mayor, M., Pepe, F., et al. 2006, Nature, 441, 305

Manabe, S., \& Wetherald, R. T. 1967, J. Atmos. Sci., 24, 241

Mlawer, E., Taubman, S., Brown, P., Iacono, M., \& Clough, S. 1997, J. Geophys. Res., 102, 16663

Pont, F., Knutson, H., Gilliland, R., Moutou, C., \& Charbonneau, D. 2008, MNRAS, 385, 109

Rauer, H., Gebauer, S., Paris, P. V., et al. 2011, A\&A, 529, A8

Rossow, W. B., \& Schiffer, R. A. 1999, Bull. Am. Met. Soc., 80, 2261

Rothman, L. S., Gordon, I. E., Barbe, A., et al. 2009, J. Quant. Spectr. Radiat. Transf., 110, 533

Schindler, T. L., \& Kasting, J. F. 2000, Icarus, 145, 262

Schreier, F., \& Schimpf, B. 2001, in IRS 2000: Current Problems in Atmospheric Radiation, eds. W. Smith, \& Y. Timofeyev (A. Deepak Publishing), 381

Segura, A., Krelove, K., Kasting, J., et al. 2003, Astrobiology, 3, 689

Segura, A., Kasting, J. F., Meadows, V., et al. 2005, Astrobiology, 5, 706

Selsis, F., Despois, D., \& Parisot, J.-P. 2002, A\&A, 388, 985

Sing, D., Pont, F., Aigrain, S., et al. 2011, MNRAS, 416, 1443

Stamnes, K., Tsay, S.-C., Wiscombe, W., \& Jayaweera, K. 1988, Appl. Opt., 27, 2502

Tinetti, G., Meadows, V., Crisp, D., et al. 2006a, Astrobiology, 6, 881

Tinetti, G., Meadows, V. S., Crisp, D., et al. 2006b, Astrobiology, 6, 34

Toon, O. B., McKay, C. P., Ackerman, T. P., \& Santhanam, K. 1989, J. Geophys. Res., 94, 16287

Udry, S., Bonfils, X., Delfosse, X., et al. 2007, A\&A, 469, 43

Vasquez, M., Gottwald, M., Gimeno García, S., et al. 2013a, Adv. Space Res., 51,835

Vasquez, M., Schreier, F., Gimeno García, S., et al. 2013b, A\&A, 549, A26

von Paris, P., Cabrera, J., Godolt, M., et al. 2011, A\&A, 534, A26

Witte, S., Helling, C., Barman, T., Heidrich, N., \& Hauschildt, P. 2011, A\&A, 529, A44

Zdunkowski, W., Trautmann, T., \& Bott, A. 2007, Radiation in the Atmosphere A Course in Theoretical Meteorology (Cambridge University Press) 